\title{
Quantum-gravity decoherence effects in neutrino oscillations: Expected constraints from CNGS and J-PARC
}

\author{
Nick E. Mavromatos, ${ }^{1}$ Anselmo Meregaglia, ${ }^{2,3}$ André Rubbia, ${ }^{2}$ Alexander S. Sakharov, ${ }^{2,4}$ and Sarben Sarkar ${ }^{1}$ \\ ${ }^{1}$ Department of Physics, King's College London, University of London, Strand, London, WC2R 2LS, United Kingdom \\ ${ }^{2}$ Swiss Institute of Technology ETH-Zürich, 8093, Zürich, Switzerland \\ ${ }^{3} I P H C$, Universit, Louis Pasteur, CNRS/IN2P3, Strasbourg, France \\ ${ }^{4}$ Theory Division, Physics Department, CERN, Geneva 23 CH 1211, Switzerland
}

(Received 15 January 2008; published 28 March 2008)

\begin{abstract}
Quantum decoherence, the evolution of pure states into mixed states, may be a feature of quantumgravity models. In most cases, such models lead to fewer neutrinos of all active flavors being detected in a long-baseline experiment as compared to three-flavor standard neutrino oscillations. We discuss the potential of the CNGS and J-PARC beams in constraining models of quantum-gravity induced decoherence using neutrino oscillations as a probe. We use as much as possible model-independent parametrizations, even though they are motivated by specific microscopic models, for fits to the expected experimental data which yield bounds on quantum-gravity decoherence parameters.
\end{abstract}

DOI: 10.1103/PhysRevD.77.053014

PACS numbers: 14.60.Pq, 04.60.- $\mathrm{m}, 14.60 . \mathrm{St}$

\section{INTRODUCTION}

If microscopic black holes, or other defects forming space-time foam, exist in the vacuum state of quantum gravity (QG) $[1,2]$, this state, in our view, will constitute an "environment" which will be characterized by some entanglement entropy, due to its interaction with lowenergy matter. Based on earlier work by Maldacena [3] on anti-de Sitter space black holes Hawking [4] has recently claimed the absence of information loss in quantum gravity. He argued that, in a superposition of topologies, the nonunitary contributions associated with the nontrivial topology decay exponentially with time, leaving only contributions to the path integral from the unitary topologically trivial configurations. However, we do not believe that this settles the issue of induced decoherence in quantum gravity since, first, the concept of anti-de Sitter spacetimes as a "regulator" has been used and, second, a Euclidean formulation of the path integral for quantum gravity has been adopted. Both these features may not be shared by an eventual true theory of quantum gravity. Furthermore, entanglement entropy is still present for an outside observer [5] and space-time foam need not consist only of black holes; other defects, e.g. pointlike D-branes, might be present. A stochastic fluctuation of populations of pointlike D-particles [6], for instance, which are known to obey infinite statistics [7] due to their infinite internal stringy states, could constitute such a decohering environment for matter propagation. There are also other quantumgravity issues that are not completely understood. In particular, it is possible that the entire concept of local effective Lagrangians breaks down in such situations.

The matter system in such a case behaves as an open quantum mechanical system, exhibiting decoherence, which has in principle detectable experimental signatures. In the context of a phenomenological parametrization of quantum-gravity induced decoherence, the first tests along these lines have been proposed in [8]. A more microscopic consideration was given in [9], where the proposed parametrization of decoherent effects of quantum gravity was forced to obey the Lindblad $[10,11]$ formalism of open systems, employing completely positive dynamical semigroup maps. This latter phenomenology, however, may not be a true feature of a quantum theory of gravity.

The decoherent approach to quantum gravity, entailing entanglement entropy, has been followed by some of the authors $[12,13]$ in many phenomenological tests or microscopic models of space-time foam [6], within the framework of noncritical string theory; the latter may be a viable (nonequilibrium) theory of space-time foam [14], based on an identification of time with the Liouville mode. The latter is viewed as a dynamical local renormalization-group scale on the world sheet of a nonconformal string. The nonconformality of the string is the result of its interaction with backgrounds which are out of equilibrium, such as those provided by twinkling microscopic black holes in the foam. The entropy in this case can be identified with the world sheet conformal anomaly of a $\sigma$-model describing the propagation of a matter string in this fluctuating background [14]. Although, within critical string theory, arguments have been given that entanglement entropy can characterize the number of microstates of anti-de Sitter black holes [15], we do not find these to be entirely conclusive, and moreover an extension of such counting to microscopic dynamical black holes, that characterize a space-time foam, is far from being understood. For instance, the process of formation and annihilation of microscopic black holes or other singular fluctuations in spacetime, including defects, may not be described by critical string theory methods.

In view of the above issues, it is evident that the debate concerning space-time foam remains open. The thermal aspects of an evaporating black hole are suggestive that the 
environment due to quantum gravity is a sort of "thermal" heat bath. This has been pursued by some authors, notably in Ref. [16]. Another proposal, the D-particle foam model [6], considers the gravitational fluctuations that could yield a foamy structure of space-time to be D-particles (pointlike stringy defects) interacting with closed strings. There are no thermal aspects but there is still the formation of horizons and entanglement entropy within a fluctuating metric framework.

In general, for phenomenological purposes, the important feature of such situations is the fact that gravitational environments, arising from space-time foam or some other, possibly semiclassical feature of QG, can still be described by nonunitary evolutions of a density matrix $\rho$. Such equations have the form

$$
\partial_{t} \rho=\Lambda_{1} \rho+\Lambda_{2} \rho
$$

where

$$
\Lambda_{1} \rho=\frac{i}{\hbar}[\rho, H]
$$

and $H$ is the Hamiltonian with a stochastic element in a classical metric. Such effects may arise from backreaction of matter within a quantum theory of gravity $[8,17]$ which decoheres the gravitational state to give a stochastic ensemble description. Furthermore within models of Dparticle foam arguments in favor of a stochastic metric have been given [12]. The Liouvillian term $\Lambda_{2} \rho$ gives rise to a nonunitary evolution. A common approach to $\Lambda_{2} \rho$, is to parametrize the Liouvillian in a so-called Lindblad form $[10,11]$ but this is not based on microscopic physics. We note at this point that any nonlinear evolutions that may characterize a full theory of QG (see e.g. a manifestation in Liouville strings [18]) can be ignored to a first approximation appropriate for the accuracy of contemporary experimental probes of QG. Generically space-time foam and the backreaction of matter on the gravitational metric may be modeled as a randomly fluctuating environment; formalisms for open quantum mechanical systems propagating in such random media can thus be applied and lead to concrete experimental predictions. The approach to these questions has to be phenomenological to some degree since QG is not sufficiently developed at a nonperturbative level.

One of the most sensitive probes of such stochastic quantum-gravity phenomena are neutrinos [13,19-27], and, in particular, high-energy ones [28,29]. It is the point of this article to study decoherence induced by nonlinear space-time foam fluctuations as a subdominant effect in neutrino oscillations at CNGS and J-PARC beams after giving an overview of the framework of decoherence phenomena in neutrino experiments.

A linear decoherence simplified model, of Lindblad type [10] has been used for the fit, following earlier work in [30]. The model of [30] involved a diagonal decoherence matrix, and in the fit of [21] decoherence was assumed to be dominant only in the antineutrino sector, in order to fit the LSND results [31] pointing towards significant $\bar{\nu}_{e} \rightarrow$ $\bar{\nu}_{\mu}$ oscillations, but suppressed oscillations in the particle sector. In this way in [21] a fit was made to a threegeneration model with the LSND "anomalous" result, without introducing a sterile neutrino. The strong $C P T$ violation in the decoherence sector, allowed for an equality of neutrino-mass differences between the two sectors, so as to be in agreement with atmospheric and solar-neutrino data. However, the LSND signal in the neutrino sector has not been corroborated by the MiniBoone collaboration [32]. This implies that, at least the simplest sterile-neutrino scenarios, suggested as a possible explanation of the LSND result, are not supported [33] in the neutrino sector by the MiniBoone experiment. However, the taking of data in the antineutrino sector is still in progress; hence the antineutrino results of the LSND remain of interest for the quantum-gravity $C P T$ violating decoherence scenarios of [21], and cannot, yet, be formally excluded by the MiniBoone collaboration.

The particular choice of [21], which yielded the best fit to all available neutrino data, involved mixed energy dependence for the (antineutrino-sector) decoherence coefficients, some of which were proportional to the neutrino energies $E$, while the rest had been inversely proportional to it, $\propto 1 / E$. In [21], the coefficients proportional to $1 / E$ were interpreted as describing ordinary matter effects, while the ones proportional to $E$ were assumed to correspond to genuine quantum-gravity effects, whose increase with the energy of the probe was consistent with the fact that the higher the (anti)neutrino energy the larger the backreaction effect on the quantum space time, and hence the larger the decoherence. The strong difference assumed in [21] between the decoherence coefficients of the particle and antiparticle sectors, although not incompatible with a breakdown of CPT at a fundamental level [13], appears at first sight somewhat curious; in fact it is unlike any other case of decoherence for other sensitive particle probes, like neutral mesons, examined in the past $[8,34]$. There, the oscillations between particle and antiparticle sectors necessitate a common decoherence environment between mesons and anti-mesons. If one accepts the universality of gravity, then the best fit of [21] seems incompatible with this property. Moreover, there are two more problematic points of the fit in [21], which were already discussed in that reference. The first point concerns the complete positivity of the model. In [30] the diagonal form of the decoherence matrix, used in [21], was taken ad hoc, without explicit mention of the necessary conditions to guarantee complete positivity, as required by the Lindblad approach. Furthermore, the particular choice of the best fit of [21] did not lead to positive-definite probabilities for the entire regime of the parameter space of the model, although the probabilities were positive definite for the portion of the parameter space appropriate for the various neutrino experiments used for the fit. Specifically, for a 
particular choice of the decoherence parameters in the (anti)neutrino sector, it was found in [21] that one obtains positive-definite transition probabilities for energies $E>$ $\mathcal{O}(1 \mathrm{MeV})$. The second, and more important point, is that the best fit of [21] is good for all the neutrino experiments available at the time, but unfortunately it could not reproduce the spectral distortion observed by the KamLand experiment [35], whose first results came out simultaneously with the results of [21]. In [25] the above weakness was rectified by requiring general conditions among the coefficients that guarantee complete positivity in the entire parameter space for the three-generation simplified Lindblad linear model of decoherence of [30], used in [21]. In fact it was shown in [25] that it was possible to choose the coefficients in a way to give a consistent and excellent fit to all available neutrino data including the spectral distortion seen by KamLand, and the LSND results [31] for the transition probabilities in the antineutrino sector.

It has been argued that quantum decoherence could be an alternative description of neutrino flavor transitions. Fits to data by different experiments such as SuperKamiokande [36] and KamLand [35] have been performed and these clearly disfavor a decoherence explanation for neutrino oscillations. However, quantum decoherence may still be a marginal effect in addition to neutrino oscillations and could give rise to damping factors in the transition probabilities reducing the number of active neutrinos being detected in a long-baseline experiment compared to what is expected from the standard neutrino oscillation scenario.

Our article is organized as follows. In Sec. II, we describe the physics of the "quantum-gravitational analogues" of the Mikheyev-Smirnov-Wolfenstein (MSW) effect and of foam models endowed with stochastic fluctuations of the space-time metric background. In Sec. III, we discuss decoherence signatures in the neutrino oscillations and review the existing bounds on these parameters using the available neutrino data, including those from KamLand [35], indicating spectral distortions. Then, in Sec. IV, we present the damping signatures and the associated fitting functions, which might be due to either the "quantum-gravitational analogue" of the MSW effect or the stochastic fluctuations of the space-time metric background. We are careful to consider various stochastic models of foam, which lead to different damping signatures, depending on the details of the underlying characteristic distribution functions [37]. In Sec. V, we estimate the sensitivity of CNGS and J-PARC experiments to the parameters of quantum-gravitational decoherence entering the set of the above-mentioned damping signatures. Finally, in Sec. VI, we compare the sensitivities estimated by means of bounds obtained from data on atmospheric, solar, and KamLand neutrino oscillations, as well as the neutral kaon system, and discuss the possible relevance of our results in guiding the construction of models of (the still elusive theory of) quantum gravity.

\section{THEORETICAL MODELS FOR QUANTUM- GRAVITY DECOHERENCE AND NEUTRINOS}

The picture we envisage is the following: there are several parallel three-brane worlds, one of which represents our observable Universe, embedded in a higherdimensional bulk, in which only gravitational (closed) string states propagate. On the brane world there are only open string states propagating, representing ordinary matter, with their ends attached on the hypersurface. Of course, there are also closed string states, either propagating along the longitudinal brane directions, or crossing the brane boundary from the bulk. As discussed in [38] consistent supersymmetric models of D-particle foam can be constructed, in which the bulk space between, say, two parallel brane worlds is populated by pointlike D-particle defects. Motion of either D-particles or branes, as required by the need to have cosmological backgrounds for a brane observer, causes supersymmetry breaking in both the brane and the bulk, and moreover results in D-particles crossing the brane boundaries. These D-particle defects can even represent compactified black holes from a fourdimensional view point, with the extra dimensions being wrapped up appropriately in Planckian size compactifications. One may then encounter a situation in which Dparticle pointlike space-time defects from the higherdimensional bulk space-time cross the three brane (where ordinary matter resides), a radically different picture from virtual excitations in a vacuum.

In Ref. [6] we have discussed the details of dynamical formation of horizons on the brane world (in the context of (Liouville) string theory), as a result of the encounter of brane matter with the crossing D-particle defect. Schematically, ordinary string matter on the brane creates - through backreaction (recoil) effects due to scattering off D-particles - sufficient distortion of space-time for dynamical horizons, surrounding the defect, to appear. The appearance of horizons in this way looks - from the point of view of a four-dimensional observer - as a dynamical "flashing on and off of a black hole," coming from the "vacuum." Using (weak) positive energy conditions, we have proven in [6] that such configurations with horizons are unstable. The lifetime of such objects is of the order of the Planck time, since this is the time uncertainty for the defect to cross the brane world and interact with stringy matter excitations. Once horizons form there is entropy production and through this irreversibility and decoherence. Consequently, such stringy black hole defects are therefore not equivalent to ordinary virtual particles in flat space-time field theories or in attempts to discuss effective local quantum-gravity approaches from the point of view of decoherence (as those mentioned in [39]).

The presence of dynamical horizons is a real effect of the ground state of quantum gravity (at least in such Liouville-string approaches to QG), which implies "real" environmental entanglement of matter systems with (gravitational) degrees of freedom behind the hori- 
zons. This leads to the problem of loss of information for particles propagating outside the horizon, and as such can lead to microscopic time irreversibility à la Wald [40], and consequent $C P T$ violation and $\mathrm{QG}$-induced decoherence. There is then a consequent nonunitary evolution of particles outside the horizon. Somewhat general arguments (even in flat space-times but with a boundary) have been put forward in the literature [41] to justify this point of view. The general message of the nonunitary evolution has then been extracted and codified with phenomenological Lindblad master equations [10,11] over two decades $[8,13,34]$ to describe particles evolving in space-time foam.

We cannot, of course, advocate at this stage that this (noncritical, Liouville) string approach, or similar, is the only consistent approach to quantum gravity. Hence, we by no means exclude the validity of the local effective approach to QG, involving only virtual gravitons; in such cases there might not be any induced decoherence [39], for reasons stated above. It is therefore a challenging experimental issue to seek such decoherence effects induced by quantum gravity, which would definitely discriminate between several models of quantum gravity.

Moreover, there is another interesting possibility regarding neutrinos. As pointed out recently in [20], the tiny mass differences between neutrino flavors may themselves (in part) be the result of a $C P T$ violating quantum-gravity background. The phenomenon, if true, would be the generalization of the celebrated MSW effect $[42,43]$. The latter arises from effective mass differences between the various neutrino flavors, as a result of different type of interactions of the various flavors with matter within the context of the standard model. The phenomenon has been generalized to randomly fluctuating media [44], which are of relevance to solar and nuclear reactor $\beta$-decays neutrinos. This stochastic MSW effect will be more relevant for us, since we consider space-time foam, as a random medium which induces flavor-sensitive mass differences. If we can extrapolate [45] semiclassical results on black-hole evaporation, in both general relativity [46] and string theory [47] to the quantum-gravity foamy ground state (assuming it exists and characterizes the ground state of some (stochastic) quantum-gravity models, it follows that microscopic black holes which are near extremal (and therefore electrically charged) would evaporate significantly less, compared with their neutral counterparts. Thus, we may assume $[20,45]$ that near extremal black holes in the foam would "live" longer, and as a result they would have more time to interact with ordinary matter, such as neutrinos. Such charged black holes would therefore constitute the dominant source of charge fluctuations in the foam that could be responsible for foam-induced neutrino-mass differences according to the idea proposed in [20]. Indeed, the emitted electrons from such black holes, which as stated above are emitted preferentially compared to muons or other charged particles, as they are the lightest, would then have more time to interact (via coherent standard model interactions) with the electron-neutrino currents, as opposed to muon neutrinos. This would create a flavor bias of the foam medium, which could then be viewed $[20,45]$ as the "quantum-gravitational analogue" of the MSW effect $[42,43]$ in ordinary media (where, again, one has only electrons, since the muons would decay quickly). In this sense, the quantum-gravity medium can be partially responsible for generating effective neutrino-mass differences [20]. As already indicated by earlier phenomenological studies [25] of quantum-gravity induced decoherence models for neutrinos, only a small part of the neutrino-mass differences and mixing can be attributed to interactions of the neutrinos with the medium of the quantum-gravity space-time foam. Nevertheless, the list of models examined so far $[20,25,45]$ is not by any means an exhaustive list. Hence, we consider the issue of the effect of quantum gravity on the size of the neutrino oscillation parameters an open one and worthy of further investigation. We also remark that in our quantumgravitational MSW scenario [20,25] the charged-black holes lead to a stochastically fluctuating medium. Consequently, we will adopt the formalism of the MSW effect for stochastically fluctuating media [44], where the density of electrons is now replaced by the density of charged-black-hole/anti-black-hole pairs.

\section{A. Quantum-gravitational MSW effect and induced decoherence}

For simplicity, we will give theoretical details for the case of two generations of neutrinos $\nu_{\mu}$ and $\nu_{\tau}$ with mass eigenvalues $m_{1}$ and $m_{2}$. We take the effective Hamiltonian to be of the form

$$
H_{\text {eff }}=H+n_{\mathrm{bh}}^{c}(r) H_{I},
$$

where $H_{I}$ is a $2 \times 2$ matrix whose entries depend on the interaction of the foam and neutrinos and $H$ is the free Hamiltonian. For the purposes of this paper we take this matrix to be diagonal in flavor space. Although we leave the entries as general constants, $a_{\nu_{i}}$, we expect them to be of the form $\propto G_{N} n_{\mathrm{bh}}^{c}(r)$; so we write $H_{I}$ as (in flavor basis)

$$
H_{I}=\left(\begin{array}{cc}
a_{\nu_{\mu}} & 0 \\
0 & a_{\nu_{\tau}}
\end{array}\right)
$$

where the foam medium is assumed to be described by Gaussian random variables [20]. We take the average number of foam particles, $\left\langle n_{\mathrm{bh}}^{c}(t)\right\rangle=n_{0}$ (a constant), and $\left\langle n_{\mathrm{bh}}^{c}(t) n_{\mathrm{bh}}^{c}\left(t^{\prime}\right)\right\rangle \sim \Omega^{2} n_{0}^{2} \delta\left(t-t^{\prime}\right)$. Following [44], we can deduce the modified time evolution of the density matrix as

$$
\frac{\partial}{\partial t}\langle\rho\rangle=-i\left[H+n_{0} H_{I},\langle\rho\rangle\right]-\Omega^{2} n_{0}^{2}\left[H_{I},\left[H_{I},\langle\rho\rangle\right]\right],
$$

where $\langle\cdots\rangle$ represents the average over the random variables of the foam. The double commutator is the $C P T$ 
violating term since although it is $C P$ symmetric it induces time irreversibility. It is also important to note that $\Lambda_{2}$ here is of the Markovian-Liouville-Lindblad form for a selfadjoint operator. This is an appropriate form for decoherence for environments about which we have little a priori knowledge. In the $C P T$ violating term we can require the density fluctuation parameter to be different for the antiparticle sector from that for the particle sector, i.e. $\bar{\Omega} \neq \Omega$, while keeping $\left\langle n_{\mathrm{bh}}^{c}(t)\right\rangle \equiv n_{0}$ the same in both sectors. Physically this means that neutrinos and antineutrinos with the same momenta, and hence interacting with the same amount of foam particles on average, will evolve differently; this is a result of $C P T$ violation.

For the remainder of this subsection we revert to an expansion in terms of mass eigenstate basis. In particular, the Hamiltonian and the density operator can be written in terms of the Pauli spin matrices $s_{\mu}$ (with $\frac{s_{0}}{2}=\mathbf{1}_{2}$ the $2 \times 2$ identity matrix) as follows:

$$
H_{\text {eff }}=\sum_{\mu=0}^{3}\left(h_{\mu}+n_{0} h_{\mu}^{\prime}\right) \frac{s_{\mu}}{2}, \quad \rho=\sum_{\nu=0}^{3} \rho_{\nu} \frac{s_{\nu}}{2}
$$

(where $H_{\text {eff }}=H+n_{0} H_{I}$ ). We find that

$$
h_{\mu}=\frac{m_{1}^{2}+m_{2}^{2}}{4 k} \delta_{\mu 0}+\frac{m_{1}^{2}-m_{2}^{2}}{2 k} \delta_{\mu 3}
$$

and

$$
\begin{aligned}
n_{0} h_{\mu}^{\prime}= & \frac{a_{\nu_{\mu}}+a_{\nu_{\tau}}}{2} \delta_{\mu 0}+\left(a_{\nu_{\mu}}-a_{\nu_{\tau}}\right) \sin 2 \theta \delta_{\mu 1} \\
& +\left(a_{\nu_{\mu}}-a_{\nu_{\tau}}\right) \cos 2 \theta \delta_{\mu 3},
\end{aligned}
$$

where $k$ is the neutrino-energy scale. The master equation in (4) then determines a matrix $\mathcal{L}_{l j}$ so that $[25,45]$

$$
\dot{\rho}_{l}=\sum_{j=1}^{3} \mathcal{L}_{l j} \rho_{j}
$$

for $l=1, \ldots, 3$. The pure state representing $\nu_{\mu}$ is given by

$$
\langle\rho\rangle^{\left(\nu_{\mu}\right)}=\frac{1}{2} \mathbf{1}_{2}+\sin (2 \theta) \frac{s_{1}}{2}+\cos (2 \theta) \frac{s_{3}}{2}
$$

and the corresponding state for $\nu_{\tau}$ is

$$
\langle\rho\rangle^{\left(\nu_{\tau}\right)}=\frac{1}{2} \mathbf{1}_{2}-\sin (2 \theta) \frac{s_{1}}{2}-\cos (2 \theta) \frac{s_{3}}{2} .
$$

If $\langle\rho\rangle(0)=\langle\rho\rangle^{\left(\nu_{\mu}\right)}$ then the probability $P_{\nu_{\mu} \rightarrow \nu_{\tau}}(t)$ of the transition $\nu_{\mu} \rightarrow \nu_{\tau}$ is given by

$$
P_{\nu_{\mu} \rightarrow \nu_{\tau}}(t)=\operatorname{Tr}\left(\langle\rho\rangle(t)\langle\rho\rangle^{\left(\nu_{\tau}\right)}\right) .
$$

In order to study decoherence we will calculate the eigenvectors $\overrightarrow{\mathfrak{e}}^{(i)}$ and corresponding eigenvalues $\lambda_{i}$ of $\mathcal{L}$ to leading order in $\Omega^{2}$. In terms of auxiliary variables $\mathcal{U}$ and $\mathcal{W}$, where

$$
\mathcal{U}=\left(a_{\nu_{\mu}}-a_{\nu_{\tau}}\right) \cos (2 \theta)+\frac{m_{1}^{2}-m_{2}^{2}}{2 k}
$$

and

$$
\mathcal{W}=\left(a_{\nu_{\mu}}-a_{\nu_{\tau}}\right) \sin (2 \theta),
$$

it is straightforward to show that

$$
\begin{aligned}
\overrightarrow{\mathrm{e}}^{(1)} & \simeq\left(\frac{\mathcal{W}}{\mathcal{U}}, 0,1\right), \\
\overrightarrow{\mathrm{e}}^{(2)} & \simeq\left(-\frac{\mathcal{U}}{\mathcal{W}},-i \frac{\sqrt{\mathcal{U}^{2}+\mathcal{W}^{2}}}{\mathcal{W}}, 1\right), \\
\overrightarrow{\mathrm{e}}^{(3)} & \simeq\left(-\frac{\mathcal{U}}{\mathcal{W}}, i \frac{\sqrt{\mathcal{U}^{2}+\mathcal{W}^{2}}}{\mathcal{W}}, 1\right),
\end{aligned}
$$

and

$$
\begin{aligned}
\lambda_{1} \simeq & -\Omega^{2}(\mathcal{W} \cos (2 \theta)-\mathcal{U} \sin (2 \theta))^{2}, \\
\lambda_{2} \simeq & -i \sqrt{\mathcal{U}^{2}+\mathcal{W}^{2}}-\frac{\Omega^{2}}{2}\left(\mathcal{U}^{2}+\mathcal{W}^{2}+(\mathcal{U} \cos (2 \theta)\right. \\
& \left.+\mathcal{W} \sin (2 \theta))^{2}\right), \\
\lambda_{3} \simeq & i \sqrt{\mathcal{U}^{2}+\mathcal{W}^{2}}-\frac{\Omega^{2}}{2}\left(\mathcal{U}^{2}+\mathcal{W}^{2}+(\mathcal{U} \cos (2 \theta)\right. \\
& \left.+\mathcal{W} \sin (2 \theta))^{2}\right) .
\end{aligned}
$$

The vector $\vec{\rho}(0)$ can be decomposed [45] as

$$
\vec{\rho}(0)=b_{1} \vec{e}^{(1)}+b_{2} \vec{e}^{(2)}+b_{2} \vec{e}^{(3)}
$$

with

$$
\mathrm{b}_{1}=\frac{\mathcal{U}^{2} \cos (2 \theta)+\mathcal{U} \mathcal{W} \sin (2 \theta)}{\mathcal{U}^{2}+\mathcal{W}^{2}}
$$

and

$$
\mathrm{b}_{2}=\frac{\mathcal{W}^{2} \cos (2 \theta)-\mathcal{U} \mathcal{W} \sin (2 \theta)}{2\left(\mathcal{U}^{2}+\mathcal{W}^{2}\right)}
$$

Hence,

$$
\rho(t)=\frac{1}{2}\left(\mathrm{~b}_{1} e^{\lambda_{1} t \vec{e}^{(1)}} \cdot \vec{s}+\mathrm{b}_{2} \vec{e}^{(2)} \cdot \vec{s}+\mathrm{b}_{2} \overrightarrow{\mathrm{e}}^{(3)} \cdot \vec{s}+\mathbf{1}_{2}\right) .
$$

From this, one can obtain from a standard analysis $[22,23,25,44]$ the following expression for the neutrino transition probability $\nu_{\mu} \leftrightarrow \nu_{\tau}$ in this case, to leading order in the small parameter $\Omega^{2} \ll 1$ : 


$$
\begin{aligned}
P_{\nu_{\mu} \rightarrow \nu_{\tau}}= & \frac{1}{2}+e^{-\Delta a_{\mu \tau}^{2} \Omega^{2} t\left(1+\left(\Delta_{12}^{2} / 4 \Gamma\right)(\cos (4 \theta)-1)\right)} \sin (t \sqrt{\Gamma}) \\
& \times \sin ^{2}(2 \theta) \Delta a_{\mu \tau}^{2} \Omega^{2} \Delta_{12}^{2}\left(\frac{3 \sin ^{2}(2 \theta) \Delta_{12}^{2}}{4 \Gamma^{5 / 2}}-\frac{1}{\Gamma^{3 / 2}}\right) \\
& -e^{-\Delta a_{\mu \tau}^{2} \Omega^{2} t\left(1+\left(\Delta_{12}^{2} / 4 \Gamma\right)(\cos (4 \theta)-1)\right)} \cos (t \sqrt{\Gamma}) \sin ^{2}(2 \theta) \\
& \times \frac{\Delta_{12}^{2}}{2 \Gamma}-e^{-\left(\left(\Delta a_{\mu \tau}^{2} \Omega^{2} t \Delta_{12}^{2} \sin ^{2}(2 \theta)\right) / \Gamma\right)} \\
& \times \frac{\left(\Delta a_{\mu \tau}+\cos (2 \theta) \Delta_{12}\right)^{2}}{2 \Gamma}
\end{aligned}
$$

where $\quad \Gamma=\left(\Delta a_{\mu \tau} \cos (2 \theta)+\Delta_{12}\right)^{2}+\Delta a_{\mu \tau}^{2} \sin ^{2}(2 \theta)$, $\Delta_{12}=\frac{\Delta m_{12}^{2}}{2 k}$, and $\Delta a_{\mu \tau} \equiv a_{\nu_{\mu}}-a_{\nu_{\tau}}$.

From (20) we easily conclude that the exponents of the damping factors due to the stochastic-medium-induced decoherence are of the generic form, for $t=L$, with $L$ the oscillation length (in units of $c=1$ ):

$$
\begin{gathered}
\text { exponent } \sim-\Delta a_{\mu \tau}^{2} \Omega^{2} t f(\theta) ; \\
f(\theta)=1+\frac{\Delta_{12}^{2}}{4 \Gamma}(\cos (4 \theta)-1), \quad \text { or } \quad \frac{\Delta_{12}^{2} \sin ^{2}(2 \theta)}{\Gamma}
\end{gathered}
$$

that is proportional to the stochastic fluctuations of the density of the medium. The reader should note at this stage that, in the limit $\Delta_{12} \rightarrow 0$, which could characterize the situation in [20], where the space-time foam effects on the induced neutrino-mass difference are the dominant ones, the damping factor is of the form exponent gravitational MSW $_{\text { }} \sim$ $-\Omega^{2}\left(\Delta a_{\mu \tau}\right)^{2} L$, with the precise value of the mixing angle $\theta$ not affecting the leading order of the various exponents. However, in that case, as follows from (20), the overall oscillation probability is suppressed by factors proportional to $\Delta_{12}^{2}$, and, hence, the stochastic gravitational MSW effect [20], although in principle capable of inducing mass differences for neutrinos, however does not suffice to produce the bulk of the oscillation probability, which is thus attributed to conventional flavor physics. The damping exponent should then be independent of the mixing angle for consistency. Indeed, we find the purely gravitational MSW to give exponent gravitational MSW $\propto \Omega^{2} \Delta^{2} L$ which is independent of $\theta$. However, this stochastic gravitational MSW effect, although capable of inducing neutrino-mass differences, gives an oscillation probability which is suppressed by factors proportional to $\Delta_{12}^{2}$. Hence, the bulk of the oscillation is due to conventional flavor physics.

After this theoretical discussion, we now proceed to give a brief description of the most important phenomenological consequences of such a scenario involving decoherence. These can help in imposing stringent constraints on the percentage of the neutrino-mass difference that could be due to the quantum-gravity medium. For simplicity we restrict ourselves to two generations, which suffices for a demonstration of the important generic properties of decoherence. The extension to three generations is straightforward, albeit mathematically more complex [25].
We note here that, for gravitationally induced MSW effects (due to, say, black-hole foam models as in $[20,45]$ )

$$
\Delta a_{\mu \tau} \propto G_{N} n_{0}
$$

with $G_{N}=1 / M_{P}^{2}, M_{P} \sim 10^{19} \mathrm{GeV}$, the four-dimensional Planck scale, and in the case of the gravitational MSW-like effect [20] $n_{0}$ represents the density of charge black-hole/ anti-black-hole pairs. This gravitational coupling replaces the weak interaction Fermi coupling constant $G_{F}$ in the conventional MSW effect. This is the case that is relevant for this work. In such a situation the density fluctuations $\Omega^{2}$ can be assumed small compared to other quantities present in the above formulas, and an expansion to leading order in $\Omega^{2}$ is appropriate.

\section{B. Stochastic fluctuations of space-time metric backgrounds}

There are other models of stochastic space-time foam also inducing decoherence, for instance the ones discussed in $[25,45]$, in which one averages over random (Gaussian) fluctuations of the background space-time metric over which the neutrino propagates. In such an approach, one considers merely the Hamiltonian of the neutrino in a stochastic metric background. The stochastic fluctuations of the metric would then pertain to the Hamiltonian (commutator) part of the density-matrix evolution. In parallel, of course, one should also consider environmental decoherence interactions of Lindblad (or other) type, which would coexist with the decoherence effects due to the stochastic metric fluctuations in the Hamiltonian. For definiteness in what follows, we restrict ourselves only to the Hamiltonian part, with the aim of demonstrating clearly the pertinent effect and study their difference from Lindblad decoherence.

In this case, one obtains transition probabilities with exponential damping factors in front of the oscillatory terms, but now the scaling with the oscillation length (time) is quadratic [25,45], consistent with time-reversal invariance of the neutrino Hamiltonian. For instance, for the two-generation case, which suffices for our qualitative purposes in this work, we may consider stochastically fluctuating space-times with metrics fluctuating along the direction of motion (for simplicity) [45]

$g^{\mu \nu}=\left(\begin{array}{cc}-\left(a_{1}+1\right)^{2}+a_{2}^{2} & -a_{3}\left(a_{1}+1\right)+a_{2}\left(a_{4}+1\right) \\ -a_{3}\left(a_{1}+1\right)+a_{2}\left(a_{4}+1\right) & -a_{3}^{2}+\left(a_{4}+1\right)^{2}\end{array}\right)$,

with random variables $\left\langle a_{i}\right\rangle=0$ and $\left\langle a_{i} a_{j}\right\rangle=\delta_{i j} \sigma_{i}$.

Two-generation Dirac neutrinos, then, which are considered for definiteness in [45] (one would obtain similar results, as far as decoherence effects are concerned in the Majorana case), with an MSW interaction $V$ (of unspecified origin, which thus could be a space-time foam effect) yield the following oscillation probability from an initial state of flavor 1 to 2 : 


$$
\operatorname{Prob}(1 \rightarrow 2)=\sum_{j, l} U_{1 j} U_{2 j}^{*} U_{1 l}^{*} U_{2 l} e^{i\left(\omega_{l}-\omega_{j}\right) t},
$$

where the time dependent part is

$$
U_{12} U_{22}^{*} U_{11}^{*} U_{21} e^{i\left(\omega_{1}-\omega_{2}\right) t}+U_{11} U_{21}^{*} U_{12}^{*} U_{22} e^{i\left(\omega_{2}-\omega_{1}\right) t}
$$

with $U$ the mixing matrix, which, in the two-flavordominance scenario we are working on here for the sake of brevity, can be parametrized by a mixing angle $\theta$ :

$$
U=\left(\begin{array}{cc}
\cos \theta & \sin \theta \\
-\sin \theta & \cos \theta
\end{array}\right) .
$$

Since the $\left\{a_{i}\right\}$ are assumed to be independent Gaussian variables, the pertinent covariance matrix $\Xi$ has the diagonal form

$$
\Xi=\left(\begin{array}{cccc}
\frac{1}{\sigma_{1}} & 0 & 0 & 0 \\
0 & \frac{1}{\sigma_{2}} & 0 & 0 \\
0 & 0 & \frac{1}{\sigma_{3}} & 0 \\
0 & 0 & 0 & \frac{1}{\sigma_{4}}
\end{array}\right),
$$

with $\sigma_{i}>0$. The calculation of transition probabilities requires the evaluation of the following average over the stochastic space-time fluctuations $a_{i}$ :

$$
\left\langle e^{i\left(\omega_{1}-\omega_{2}\right) t}\right\rangle \equiv \int d^{4} a \exp (-\vec{a} \cdot \Xi \cdot \vec{a}) e^{i\left(\omega_{1}-\omega_{2}\right) t} \frac{\operatorname{det} \Xi}{\pi^{2}} .
$$

The result is [45]

$$
\begin{aligned}
\left\langle e^{i\left(\omega_{1}-\omega_{2}\right) t}\right\rangle= & e^{i\left(\left(\left(z_{0}^{+}-z_{0}^{-}\right) t\right) / k\right)} e^{-(1 / 2)\left(-i \sigma_{1} t\left(\left(\left(m_{1}^{2}-m_{2}^{2}\right) / k\right)+V \cos 2 \theta\right)\right)} e^{-(1 / 2)\left(\left(\left(i \sigma_{2} t\right) / 2\right)\left(\left(\left(m_{1}^{2}-m_{2}^{2}\right) / k\right)+V \cos 2 \theta\right)-\left(\left(i \sigma_{3} t\right) / 2\right) V \cos 2 \theta\right)} \\
& \times e^{-\left(\left(\left(\left(m_{1}^{2}-m_{2}^{2}\right)^{2}\right) / 2 k^{2}\right)\left(9 \sigma_{1}+\sigma_{2}+\sigma_{3}+\sigma_{4}\right)+\left(\left(2 V \cos 2 \theta\left(m_{1}^{2}-m_{2}^{2}\right)\right) / k\right)\left(12 \sigma_{1}+2 \sigma_{2}-2 \sigma_{3}\right)\right) t^{2}},
\end{aligned}
$$

where again $k$ is the neutrino-energy scale, $\sigma_{i}, i=1, \ldots 4$ parametrize appropriately the stochastic fluctuations of the metric in the model of [45], $Y=\frac{V k}{m_{1}^{2}-m_{2}^{2}},|Y| \ll 1$, and $k^{2} \gg m_{1}^{2}, m_{2}^{2}$, and

$$
\begin{aligned}
z_{0}^{+}= & \frac{1}{2}\left(m_{1}^{2}+\Upsilon(1+\cos 2 \theta)\left(m_{1}^{2}-m_{2}^{2}\right)\right. \\
& \left.+\Upsilon^{2}\left(m_{1}^{2}-m_{2}^{2}\right) \sin ^{2} 2 \theta\right) \\
z_{0}^{-}= & \frac{1}{2}\left(m_{2}^{2}+\Upsilon(1-\cos 2 \theta)\left(m_{1}^{2}-m_{2}^{2}\right)\right. \\
& \left.-\Upsilon^{2}\left(m_{1}^{2}-m_{2}^{2}\right) \sin ^{2} 2 \theta\right) .
\end{aligned}
$$

Note that the metric fluctuations- $\sigma_{i}$ induced modifications of the oscillation period, as well as exponential $e^{-(. .) t^{2}}$ time-reversal invariant damping factors [45], in contrast to the Lindblad decoherence, in which the damping was of the form $e^{-(\ldots) t}$. At first thought, one may attribute this feature to the fact that, in this approach, only the Hamiltonian terms are taken into account (in a stochastically fluctuating metric background), and as such timereversal invariance $t \rightarrow-t$ is not broken explicitly. But there is of course decoherence, and the associated damping.

However, upon closer inspection things are not as simple. As shown in [37], the power of the time variable $t$ in the associated damping is crucially dependent on the type of the distribution characterizing the gravitational fluctuations. In terms of our D-particle-recoil induced stochastic model of space-time foam $[6,12,45]$, such distributions characterize the ensemble of velocities of the gas of Dparticles involved in the foam, with which the neutrinos interact, and which in turn affects the induced metric fluctuations, as explained above. Assume, for instance, that the distribution of the (induced) metric fluctuations are of Cauchy-Lorentz type, which could be induced by a distribution of D-particle velocities in a D-foam model of the type considered in [38]. Such a distribution has unde- fined mean and variance as well as undefined or infinite higher moments. Assuming for concreteness a case with zero mean (in the sense of principal values), the pertinent distribution function is taken to be [37]

$$
f(x)=\frac{\xi}{x^{2}+\xi^{2}}
$$

with $\xi>0$ the characteristic scale parameter of the Cauchy-Lorentz distribution. In that case, the pertinent statistical average of the associated oscillation probability for a two-flavor neutrino problem would be given, to leading order in an expansion in powers of $m_{i} / k$, of interest to us here, by

$$
\left\langle e^{i\left(\omega_{1}-\omega_{2}\right) t}\right\rangle_{\text {C.L. }} \simeq \exp (i k t \Delta-\xi k t|\Delta|), \quad \Delta=\frac{m_{1}^{2}-m_{2}^{2}}{2 k^{2}} .
$$

From the above equation we do observe a linear damping, similar to the Lindblad environment case. The important point to notice is that in this case, the damping exponent is of order

$$
\exp \left(-\Omega_{\mathrm{CL}}^{2} t\right), \quad \text { with } \Omega_{\mathrm{CL}}^{2} \simeq \xi \frac{\left|m_{1}^{2}-m_{2}^{2}\right|}{2 k} .
$$

The reader should compare the linear power of the (small) quantity $\left|m_{1}^{2}-m_{2}^{2}\right| / k$ entering the damping exponent (29) in the Cauchy-Lorentz stochastic foam model to the quadratic power of that quantity entering the Gaussian model of foam (25), where the pertinent exponents are proportional to factors of the form $\left(m_{1}^{2}-m_{2}^{2}\right)^{2} / k^{2}$ and hence much smaller, provided of course the parameters $\sigma^{2}$ and $\xi$ are of similar order. At this stage, these are treated as phenomenological parameters, since their order depends on the details of the underlying model. For instance, in the case of the D-particle foam model [6,38], such information depends on the dynamics of the gas of bulk D-particles, 
which probably is an issue that can only be resolved within a microscopic M-theory model.

At this stage we would like to draw the reader's attention to a possible interpretation [29] of the Lindblad-type exponential damping (29) of the Cauchy-Lorentz distribution with $1 / E$ dependence as a neutrino decay,

$$
\exp \left(-\Omega_{\text {C.L. }}^{2} t\right) \equiv \exp \left(-t / \tau_{\text {lab }}\right)=\exp \left(-t m_{\nu_{i}} / E \tau_{\text {rest }}\right),
$$

from which we can get a lower limit on the (unstable) neutrinos lifetimes. We shall come back to this issue in the concluding section of the article, when we provide the relevant experimental bounds.

It must be stressed at this point, before closing this subsection, that the above considerations, especially the ones concerning the form and order of the decoherence damping factors, of interest to us in this work, although derived in a two-dimensional toy model (considering metric deformations primarily along the direction of motion of the neutrino probe), nevertheless are valid qualitatively in a full fledged four space-time dimensional model. This has been demonstrated in [37], where realistic models of neutrinos propagating in four-dimensional, stochastically fluctuating, space-time backgrounds have been considered in detail, with results similar to the ones considered in [12] and reviewed above.

\section{Mimicking decoherence via conventional uncertainties in neutrino energy and oscillation length}

A few remarks are now in order regarding the similarity of this latter type of decoherence (25) with the one mimicked [48] by ordinary uncertainties in neutrino experiments over the precise energy $E$ of the beam (and in some cases over the oscillation length $L$ ). Indeed, consider the Gaussian average of a generic neutrino oscillation probability over the $L / E$ dependence $\langle P\rangle=\int_{-\infty}^{\infty} d x P(x) \times$ $\frac{1}{\sigma \sqrt{2 \pi}} e^{-\left((x-l)^{2} / 2 \sigma^{2}\right)}$, with $l=\langle x\rangle$ and $\sigma=\sqrt{\left\langle(x-\langle\bar{x}\rangle)^{2}\right\rangle}$, $x=\frac{L}{4 E}$, and assuming the independence of $L$ and $E$, which allows one to write $\langle L / E\rangle=\langle L\rangle /\langle E\rangle$. A pessimistic and an optimistic upper bound for $\sigma$ are given by [48]

(i) pessimistic: $\sigma \simeq \Delta x=\Delta \frac{L}{4 E} \leq \Delta L\left|\frac{\partial x}{\partial L}\right|_{L=\langle L\rangle, E=\langle E\rangle}+$

$$
\Delta E\left|\frac{\partial x}{\partial E}\right|_{L=\langle L\rangle, E=\langle E\rangle}=\frac{\langle L\rangle}{4\langle E\rangle}\left(\frac{\Delta L}{\langle L\rangle}+\frac{\Delta E}{\langle E\rangle}\right)
$$

(ii) optimistic: $\sigma \leq \frac{\langle L\rangle}{4\langle E\rangle} \sqrt{\left(\frac{\Delta L}{\langle L\rangle}\right)^{2}+\left(\frac{\Delta E}{\langle E\rangle}\right)^{2}}$.

Then, it is easy to arrive at the expression [48]

$$
\begin{aligned}
\left\langle P_{\alpha \beta}\right\rangle= & \delta_{\alpha \beta}-2 \sum_{a=1}^{n} \sum_{b=1, a<b}^{n} \operatorname{Re}\left(U_{\alpha a}^{*} U_{\beta a} U_{\alpha b} U_{\beta b}^{*}\right) \\
& \times\left(1-\cos \left(2 \ell \Delta m_{a b}^{2}\right) e^{-2 \sigma^{2}\left(\Delta m_{a b}^{2}\right)^{2}}\right) \\
& -2 \sum_{a=1}^{n} \sum_{b=1, a<b}^{n} \operatorname{Im}\left(U_{\alpha a}^{*} U_{\beta a} U_{\alpha b} U_{\beta b}^{*}\right) \\
& \times \sin \left(2 \ell \Delta m_{a b}^{2}\right) e^{-2 \sigma^{2}\left(\Delta m_{a b}^{2}\right)^{2}} \quad \text { with } \ell \equiv \frac{\langle L\rangle}{4\langle E\rangle}
\end{aligned}
$$

with $U$ the appropriate mixing matrix. Notice the $\sigma^{2}$ damping factor of neutrino oscillation probabilities, which has the similar form in terms of the oscillation-length dependence ( $L^{2}$ dependence) as the corresponding damping factors due to the stochasticity of the space-time background in (25). It is noted, however, that here $l$ has to do with the sensitivity of the experiment, and thus the physics is entirely different.

In the case of space-time stochastic backgrounds, one could still have induced uncertainties in $E$ and $L$, which however are of fundamental origin, and are expected to be more suppressed than the uncertainties due to ordinary physics, described above. Apart from their magnitude, their main difference from the uncertainties in (31) has to do with the specific dependence of the corresponding $\sigma^{2}$ in that case on both $E$ and $L$. For generic space-time foam models, it is expected that an uncertainty in $E$ or $L$ due to the "fuzziness" of space-time at a fundamental (Planckian) level will increase with the energy of the probe, $\delta E / E, \delta L / L \propto\left(E / M_{P}\right)^{\alpha}, \alpha>0$, since the higher the energy the bigger the disturbance (and hence backreaction) on the space-time medium. In contrast, ordinary matter effects decrease with the energy of the probe $[48,49]$.

\section{PREVIOUS DECOHERENT FITS WITH EXISTING NEUTRINO DATA AND PHYSICAL INTERPRETATION}

The first complete phenomenological attempt to fit decoherence models to atmospheric neutrino data was done in [19], where for simplicity a two-generation neutrino model with completely positive Lindblad decoherence, characterized by a single parameter $\gamma$, and leading to exponential damping with time of the relevant oscillatory terms in the respective oscillation probabilities, was considered.

Various dependencies on the energy $E$ of the neutrino probes have been assumed, in a phenomenological fashion, for the Lindblad decoherence coefficient $\gamma=\gamma_{\mathrm{Lnb}}\left(\frac{E}{\mathrm{GeV}}\right)^{n}$, with $n=0,2,-1$. The sensitivities in the work of [19] from atmospheric neutrinos (plus accelerator data [26]) at $90 \%$ C.L. can be summarized by the following bounds on the parameter $\gamma_{\mathrm{Lnb}}$ :

$$
\begin{array}{ll}
\gamma_{\mathrm{Lnb}}<0.4 \times 10^{-22} \mathrm{GeV}, & n=0 \\
\gamma_{\mathrm{Lnb}}<0.9 \times 10^{-27} \mathrm{GeV}, & n=2 \\
\gamma_{\text {Lnb }}<0.7 \times 10^{-21} \mathrm{GeV}, & n=-1 .
\end{array}
$$

Recently [27], updated values on these parameters, referred to $95 \%$ C.L., have been provided by means of combining solar-neutrino and KamLand data: 


$$
\begin{array}{lll}
\gamma_{\text {Lnb }}<0.67 \times 10^{-24} \mathrm{GeV}, & & n=0 \\
\gamma_{\text {Lnb }}<0.47 \times 10^{-20} \mathrm{GeV}, & & n=2 \\
\gamma_{\text {Lnb }}<0.78 \times 10^{-26} \mathrm{GeV}, & & n=-1 .
\end{array}
$$

It should be remarked that all these bounds should be taken with a grain of salt, since there is no guarantee that in a theory of quantum gravity $\gamma_{\mathrm{Lnb}}$ should be the same in all channels, or that the functional dependence of the decoherence coefficients $\gamma$ on the probe's energy $E$ follows a simple power law. Complicated functional dependencies $\gamma(E)$ might be present.

We shall come back to these bounds in the discussion section of the article, when we compare the potential of upcoming neutrino data, with energies of order of tens of $\mathrm{GeV}$, from CNGS facility. We also investigate the sensitivity of the experiments at J-PARC. The J-PARC beam operates at rather lower energies comparing to CNGS; however, the fact that the maximum of oscillation in the spectrum will be measured by $\mathrm{T} 2 \mathrm{~K}$ experiment allows one to achieve a remarkable sensitivity to those damping exponents with low power energy dependence, as compared with $[19,27]$. The CNGS is very sensitive to the $E^{2}$ dependent case despite the fact that the spectrum of atmospheric neutrinos used in [19] spans a wide range of energy which extends to $100-1000 \mathrm{GeV}$. The $E^{2}$ dependence, for instance, could characterize Cauchy-Lorentz stochastic models of space-time foam.

In [25] a three-generation Lindblad decoherence model of neutrinos has been compared against all available at the time experimental data, taking into account the recent results from the KamLand experiment [35] indicating spectral distortions.

The results are summarized in Fig. 1, which demonstrates the agreement (left) of the model with the KamLand spectral distortion data [35], and the best fit (right) for the Lindblad decoherence model used in Ref. [25].

The best fit has the feature that only some of the oscillation terms in the three-generation probability formula have nontrivial damping factors, with their exponents being independent of the oscillation length, specifically [25]. If we denote those nontrivial exponents as $\mathcal{D} \cdot L$, we obtain from the best-fit of [25]:

$$
\mathcal{D}=-\frac{1.3 \times 10^{-2}}{L},
$$

in units of $1 / \mathrm{km}$ with $L=t$ the oscillation length. The $1 / L$ behavior of $\mathcal{D}_{11}$ implies, as we mentioned, oscillationlength independent Lindblad exponents.

In [25] an analysis of the two types of the theoretical models of space-time foam, discussed in Sec. II, has been performed in light of the result of the fit (34). The conclusion was that the model of the stochastically fluctuating media (20) (extended appropriately to three generations [25], so as to be used for comparison with the real data) cannot provide the full explanation for the fit, for the following reason: if the decoherent result of the fit (34) was exclusively due to this model, then the pertinent decoherent coefficient in that case, for, say, the KamLand experiment with an $L \sim 180 \mathrm{Km}$, would be $|\mathcal{D}|=$ $\Omega^{2} G_{N}^{2} n_{0}^{2} \sim 2.84 \times 10^{-21} \mathrm{GeV}$ (note that the mixing angle part does not affect the order of the exponent). Smaller values are found for longer $L$, such as in atmospheric neutrino experiments. The independence of the relevant damping exponent from the oscillation length, then, as required by (34), may be understood as follows in this context: In the spirit of [20], the quantity $G_{N} n_{0}=\xi \frac{\Delta m^{2}}{E}$, where $\xi \ll 1$ parametrizes the contributions of the foam to the induced neutrino-mass differences, according to our discussion above. Hence, the damping exponent becomes in this case $\xi^{2} \Omega^{2}\left(\Delta m^{2}\right)^{2} \cdot L / E^{2}$. Thus, for oscillation
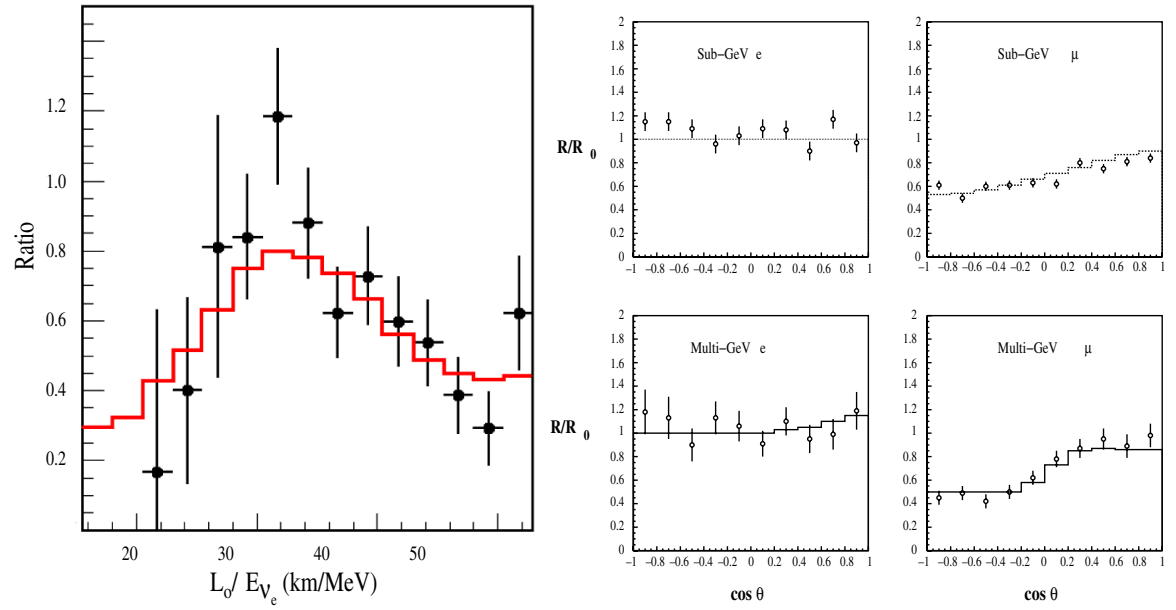

FIG. 1 (color online). Left: Ratio of the observed $\bar{\nu}_{e}$ spectrum to the expectation versus $L_{0} / E$ for our decoherence model. The dots correspond to KamLand data. Right: Decoherence fit. The dots correspond to Super-Kamiokande data. 
lengths $L$ we have $L^{-1} \sim \Delta m^{2} / E$, and one is left with the following estimate for the dimensionless quantity $\xi^{2} \Delta m^{2} \Omega^{2} / E \sim 1.3 \times 10^{-2}$. This implies that the quantity $\Omega^{2}$ is proportional to the probe energy $E$. In principle, this is not an unreasonable result, and it is in the spirit of [20], since backreaction effects onto space-time, which affect the stochastic fluctuations $\Omega^{2}$, are expected to increase with the probe energy $E$. However, due to the smallness of the quantity $\Delta m^{2} / E$, for energies of the order of $\mathrm{GeV}$, and $\Delta m^{2} \sim 10^{-3} \mathrm{eV}^{2}$, we conclude (taking into account that $\xi \ll 1$ ) that $\Omega^{2}$ in this case would be unrealistically large for a quantum-gravity effect in the model.

We remark at this point that, in such a model, we can in principle bound independently the $\Omega$ and $n_{0}$ parameters by looking at the modifications induced by the medium in the arguments of the oscillatory functions of the probability (20), that is the period of oscillation. Unfortunately this is too small to be detected in the above example, for which $\Delta a_{e \mu} \ll \Delta_{12}$.

The second model (25) of stochastic space-time can also be confronted with the data, since in that case (34) would imply for the pertinent damping exponent

$$
\begin{aligned}
& \left(\frac{\left(m_{1}^{2}-m_{2}^{2}\right)^{2}}{2 k^{2}}\left(9 \sigma_{1}+\sigma_{2}+\sigma_{3}+\sigma_{4}\right)+\frac{2 V \cos 2 \theta\left(m_{1}^{2}-m_{2}^{2}\right)}{k}\right. \\
& \left.\quad \times\left(12 \sigma_{1}+2 \sigma_{2}-2 \sigma_{3}\right)\right) t^{2} \sim 1.3 \times 10^{-2}
\end{aligned}
$$

Ignoring subleading MSW effects $V$, for simplicity, and considering oscillation lengths $t=L \sim \frac{2 k}{\left(m_{1}^{2}-m_{2}^{2}\right)}$, we then observe that the independence of the length $L$ result of the experimental fit, found above, may be interpreted, in this case, as bounding the stochastic fluctuations of the metric to $9 \sigma_{1}+\sigma_{2}+\sigma_{3}+\sigma_{4} \sim 1.3 \times 10^{-2}$. Again, this is too large to be a quantum-gravity effect, which means that the $L^{2}$ contributions to the damping due to stochastic fluctuations of the metric, as in the model of [45] above (25), cannot be the exclusive explanation of the fit.

The analysis of [25] also demonstrated that, at least as far as an order of magnitude of the effect is concerned, a reasonable explanation of the order of the damping exponent (34) is provided by Gaussian-type energy fluctuations, due to ordinary physics effects, leading to decoherencelike damping of oscillation probabilities of the form (31). The order of these fluctuations, consistent with the independence of the damping exponent on $L$ (irrespective of the power of $L$ ), is

$$
\frac{\Delta E}{E} \sim 1.6 \times 10^{-1},
$$

if one assumes that this is the principal reason for the result of the fit.

However, not even this can be the end of the story, given that the result (34) pertains only to some of the oscillation terms and not all of them, which would be the case expected for the ordinary physics uncertainties (31). The fact that the best-fit model includes terms which are not suppressed at all calls for a more radical explanation of the fit result, and the issue is still wide open.

It is interesting, however, that the current neutrino data can already impose stringent constraints on quantumgravity models, and exclude some of them from being the exclusive source of decoherence, as we have discussed above. Of course, this is not a definite conclusion because one cannot exclude the possibility of other classes of theoretical models of quantum gravity, which could escape these constraints. At present, however, we are not aware of any such theory. We would like now to revisit the above constraints in upcoming neutrino data from the experiments at the CNGS and J-PARC facilities. This is discussed in the next sections.

\section{THE COMBINED FIT TO QUANTUM-GRAVITY DECOHERENCE SIGNATURES}

In the previous sections we have discussed several theoretical models of quantum-gravity-induced decoherence independently, assuming each time only one dominant type of decoherence: (i) Lindblad-type, through the representation of the quantum-gravity space-time foam as a stochastic medium, (20), (ii) stochastically fluctuating space-time backgrounds, (25), and (iii) induced decoherencelike evolution, as a result of uncertainties in the energy and/or oscillation lengths of the neutrinos (31).

The various types of decoherence can be mainly distinguished by the form of their exponential damping factor, as far as the power of the oscillation length $L$ in the exponent is concerned, and the associated energy dependence [50]. Model-independent data fits should combine, in general, the various types of decoherence-deformed oscillations, given that dominance of one or the other type may not be necessarily a feature of a quantum-gravity model.

It is the purpose of this section, and one of the main objectives of this work, to establish the limit of sensitivity of CNGS and J-PARC beams, in a model-independent way, to a simple parametrization of the above effects, combined in a single model for oscillations between flavors $a, b=$ $1 \ldots n$ of the form

$$
\begin{aligned}
\left\langle P_{\alpha \beta}\right\rangle= & \delta_{\alpha \beta}-2 \sum_{a=1}^{n} \sum_{b=1, a<b}^{n} \operatorname{Re}\left(U_{\alpha a}^{*} U_{\beta a} U_{\alpha b} U_{\beta b}^{*}\right) \\
& \times\left(1-\cos \left(2 \ell \Delta m_{a b}^{2}\right) e^{-q_{1} L-q_{2} L^{2}}\right) \\
& -2 \sum_{a=1}^{n} \sum_{b=1, a<b}^{n} \operatorname{Im}\left(U_{\alpha a}^{*} U_{\beta a} U_{\alpha b} U_{\beta b}^{*}\right) \\
& \times \sin \left(2 \ell \Delta m_{a b}^{2}\right) e^{-q_{1} L-q_{2} L^{2}} \quad \text { with } \ell \equiv \frac{L}{4 E},
\end{aligned}
$$

where $L \simeq t$ (in units of $c=1$ ) is the oscillation length. In general one may parametrize the damping exponents by polynomials in $L$ [50] of any degree, but parametrizations of degrees higher than 2 are not favored by the class of 
quantum-gravity decoherence models considered in the literature so far $[13,45]$, and reviewed above.

From (20) and (25), we observe that (37) is oversimplified in that it ignores possible modifications of the oscillation period, which do exist in various microscopic models as a result of the decoherence or stochasticmedium effects. A complete theoretical treatment requires solving the evolution equations for the reduced density matrix of neutrinos in a combined situation involving simultaneously stochastic fluctuations of the background space-time metric and interactions (of Lindblad type) with a stochastically fluctuating quantum-space-time medium. This will be left for future work. However, for our purposes in the current article, we note that it is a reasonable assumption that such modifications to the oscillation period are suppressed as compared with the ordinary oscillation terms, and as such the dominant, modelindependent, terms appear to be only the exponents of the damping factors. Concerning the latter, we also observe from (20) that, in general, there are slight differences among the various exponents accompanying the oscillation terms in stochastic-medium models, which however are all of the same order of magnitude, and hence the error one makes in assuming the simplifying two-parameter $\left(q_{1}, q_{2}\right)$ damping decoherence form (37) is negligible.

For our phenomenological purposes in this work, therefore, the only important point to notice is that the parameters $q_{i}, i=1,2$ may be in general energy dependent, expressing backreaction effects of the (neutrino) matter onto the fluctuating space-time. Following earlier treatments and theoretical quantum-gravity-decoherence models $[13,19]$, we shall consider the following three cases of generic energy dependence of the decoherence coefficients $q_{i}, i=1,2$ :

$$
q_{i}, \quad i=1,2 \propto E^{n}, \quad n=-1,0,2,
$$

where the reader should have in mind that in each case the pertinent decoherence coefficient has the appropriate units, as being a dimensionful quantity.

For our studies we use two sets of the one and two parametric models covering the main variety of phenomenologies for quantum-gravity-induced decoherence phenomena described by the expression (37). The first set of the models under consideration concerns the presence of linear Lindblad-type mapping operator in the equation for the evolution of the density matrix for the pure neutrino quantum states $[13,19,22,23,26,48]$. The oscillation probabilities corrected for the decoherence effects with different energy dependence in the exponentials read

(i) no neutrino-energy dependence

$$
\begin{aligned}
P_{\nu_{\mu} \rightarrow \nu_{\tau}}= & \frac{1}{2} \sin ^{2}\left(2 \theta_{23}\right)\left[1-\exp \left(-5 \times 10^{9} \gamma_{0} L\right)\right. \\
& \left.\times \cos \left(\frac{2.54 \Delta m^{2}}{E} L\right)\right]
\end{aligned}
$$

(ii) inversely proportional to the neutrino energy (e.g. the case of Cauchy-Lorentz type of stochastic foam $[29,37])$

$$
\begin{aligned}
P_{\nu_{\mu} \rightarrow \nu_{\tau}}= & \frac{1}{2} \sin ^{2}\left(2 \theta_{23}\right)\left[1-\exp \left(\frac{-2.54 \gamma_{-1}^{2} L}{E}\right)\right. \\
& \left.\times \cos \left(\frac{2.54 \Delta m^{2}}{E} L\right)\right]
\end{aligned}
$$

(iii) proportional to the neutrino energy squared

$$
\begin{aligned}
P_{\nu_{\mu} \rightarrow \nu_{\tau}}= & \frac{1}{2} \sin ^{2}\left(2 \theta_{23}\right)\left[1-\exp \left(-5 \times 10^{27} \gamma_{2} E^{2} L\right)\right. \\
& \left.\times \cos \left(\frac{2.54 \Delta m^{2}}{E} L\right)\right]
\end{aligned}
$$

where $\gamma_{0}, \gamma_{-1}^{2}$, and $\gamma_{2}$ are measured in $\mathrm{eV}, \mathrm{eV}^{2}$ length, and $\mathrm{eV}^{-1}$, respectively, the mass square difference $\Delta m^{2}$ is measured in $\mathrm{eV}^{2}$, the energy $E$ is measured in $\mathrm{GeV}$; and the path, $L$, is measured in $\mathrm{km}$.

The second set of the models concerns the gravitational MSW stochastic effect (20) with linear and quadratic (25) time dependent fluctuations of space-time foam described by

$$
\begin{aligned}
P_{\nu_{\mu} \rightarrow \nu_{\tau}}= & \frac{1}{2}-\exp \left(-\kappa_{1}\right) \frac{\cos ^{2}\left(2 \theta_{23}\right)}{2}-\frac{1}{2} \exp \left(-\kappa_{2}\right) \\
& \times \cos \left(\frac{2.54 \Delta m^{2}}{E} L\right) \sin ^{2}\left(2 \theta_{23}\right),
\end{aligned}
$$

where the exponential damping factors are chosen as

(i) no energy dependence, with linear

$$
\begin{aligned}
& \kappa_{1}=5 \times 10^{9} \alpha^{2} L \sin ^{2}(2 \theta) ; \\
& \kappa_{2}=5 \times 10^{9} \alpha^{2} L(1+0.25(\cos (4 \theta)-1))
\end{aligned}
$$

quadratic

$$
\begin{aligned}
& \kappa_{1}=2.5 \times 10^{19} \alpha_{1}^{2} L^{2} \sin ^{2}(2 \theta) ; \\
& \kappa_{2}=2.5 \times 10^{19} \alpha_{1}^{2} L^{2}(1+0.25(\cos (4 \theta)-1))
\end{aligned}
$$

and combined time evolution

$$
\begin{aligned}
\kappa_{1}= & \left(5 \times 10^{9} \gamma_{1}^{2} L+2.5 \times 10^{19} \gamma_{2}^{2} L^{2}\right) \sin ^{2}(2 \theta) \\
\kappa_{2}= & \left(5 \times 10^{9} \gamma_{1}^{2} L+2.5 \times 10^{19} \gamma_{2}^{2} L^{2}\right) \\
& \times(1+0.25(\cos (4 \theta)-1))
\end{aligned}
$$

(ii) proportional to the neutrino energy, with linear

$$
\begin{aligned}
& \kappa_{1}=5 \times 10^{18} \beta^{2} E L \sin ^{2}(2 \theta) \\
& \kappa_{2}=5 \times 10^{18} \beta^{2} E L(1+0.25(\cos (4 \theta)-1))
\end{aligned}
$$

quadratic 


$$
\begin{aligned}
& \kappa_{1}=2.5 \times 10^{28} \beta_{2}^{2} E L^{2} \sin ^{2}(2 \theta) ; \\
& \kappa_{2}=2.5 \times 10^{28} \beta_{2}^{2} E L^{2}(1+0.25(\cos (4 \theta)-1))
\end{aligned}
$$

and combined time evolution

$$
\begin{aligned}
\kappa_{1}= & \left(5 \times 10^{18} \gamma_{1}^{\prime 2} E L+2.5 \times 10^{28} \gamma_{2}^{\prime 2} E L^{2}\right) \sin ^{2}(2 \theta) \\
\kappa_{2}= & \left(5 \times 10^{18} \gamma_{1}^{\prime 2} E L+2.5 \times 10^{28} \gamma_{2}^{\prime 2} E L^{2}\right) \\
& \times(1+0.25(\cos (4 \theta)-1))
\end{aligned}
$$

(iii) proportional to the neutrino energy squared, with linear time evolution

$$
\begin{aligned}
& \kappa_{1}=5 \times 10^{27} \beta_{1}^{2} E^{2} L \sin ^{2}(2 \theta) ; \\
& \kappa_{2}=5 \times 10^{27} \beta_{1}^{2} E^{2} L(1+0.25(\cos (4 \theta)-1))
\end{aligned}
$$

The energy and the path length in (43)-(49) are measured in $\mathrm{GeV}$ and $\mathrm{km}$, respectively, while the parameters in damping exponentials are given in $\mathrm{eV}$ in respective power (see Table I for details).

\section{SENSITIVITY OF CNGS AND J-PARC BEAMS TO QUANTUM-GRAVITY DECOHERENCE}

In this section we study the expected sensitivity of the CNGS and J-PARC beams to the quantum-gravitational decoherence phenomena described by (39)-(49), considering them as subdominant contributions to the atmospheric oscillations effects.

Both CNGS and J-PARC are conventional neutrino beams where neutrinos are produced by the decay of secondary particles (pions and kaons) obtained from the collision of the primary proton on a graphite target. For the CNGS beam, the protons come from the CERN-SPS facility with a momentum of $400 \mathrm{GeV} / c$, whereas in the case of the J-PARC [51] the protons are produced in Tokai (Japan) and have a momentum of $40 \mathrm{GeV} / c$. The expected number of protons on target (p.o.t.) per year at the nominal intensity is $4.5 \times 10^{19}$ and $1 \times 10^{21}$, respectively, for the CNGS and J-PARC beam and the envisaged run length is 5 years in both cases.

Both beams will be used for long-baseline neutrino experiments which, starting from a $\nu_{\mu}$ beam, will search for neutrino oscillations. The OPERA experiment will measure neutrino events on the CNGS beam using a 2 kton detector which relies on the photographic emulsion technique, located at a baseline of $732 \mathrm{~km}$; the first neutrino events were observed in August 2006 [52].

The T2K experiment will use the J-PARC beam measuring neutrino events with the Super-Kamiokande [53] detector (a water Cherenkov detector with an active volume of $22.5 \mathrm{kton}$ ) at a baseline of $295 \mathrm{~km}$.
Although CNGS beam designed in a way to be optimized for the $\nu_{\mu} \rightarrow \nu_{\tau}$ oscillation searches through the detection of $\tau$ lepton production in a pure $\nu_{\mu}$ beam, there is also a possibility to measure $\nu_{\mu}$ spectrum by reconstructing $\mu$ from the charged current (CC) events caused by $\nu_{\mu}$. Moreover, for this experiment, we can take advantage of the high mean value for the energy of $\nu_{\mu}$ s which makes the exponential damping factors more pronounced for some cases described in the previous section.

The number of $\mu$ is given by the convolution of the $\nu_{\mu}$ flux $d \phi_{\nu_{\mu}} / d E$ with the $\nu_{\mu}$ CC cross section on lead $\sigma_{\nu_{\mu}}^{\mathrm{CC}}(E)$, weighted by the $\nu_{\mu} \rightarrow \nu_{\mu}$ surviving probability $P_{\nu_{\mu} \rightarrow \nu_{\mu}}$, times the efficiency $\epsilon_{\mu \mu}$ of muon reconstruction of a given detector:

$$
\frac{d N_{\mu \mu}}{d E}=A_{\mu \mu} \frac{d \phi_{\nu_{\mu}}}{d E} P_{\nu_{\mu} \rightarrow \nu_{\mu}} \sigma_{\nu_{\mu}}^{\mathrm{CC}}(E) \epsilon_{\mu \mu},
$$

where $A_{\mu \mu}$ is a normalization factor which takes into account the target mass and the normalization of the $\nu_{\mu}$ in physical units. In our study we assumed an overall efficiency $\epsilon_{\mu \mu}$ of $93.5 \%$ for the OPERA experiment and of $90 \%$ for the $\mathrm{T} 2 \mathrm{~K}$ one as stated in the experiment proposals.

To estimate quantitatively the sensitivity of CNGS on $P_{\nu_{\mu} \rightarrow \nu_{\tau}}$ described by (39)-(49), we simulated the theoretical spectra of the reconstructed $\nu_{\mu}$ events for various values of damping parameters. Since there is no near detector at the neutrino source, the overall normalization of the unoscillated neutrino flux cannot be controlled with the precision better than $20 \%$, therefore such a normalization has been taken into account in our $\chi^{2}$ analysis to estimate the expected limits of sensitivity on the damping parameters:

$$
\chi^{2}=\sum_{i}\left[x_{i}-a P_{i}\right]^{2} / \sigma_{i}^{2}+(1-a)^{2} / \tilde{\sigma}^{2},
$$

where $x_{i}$ is the expected number of $\nu_{\mu} \mathrm{CC}$ events contained in the $i$ th energy bin considering standard three flavor oscillation, $P_{i}$ is the number of events in the $i$ th bin theoretically expected when some decoherence parameters are considered, and $\sigma_{i}$ represents the error on the number of events in the $i$ th bin. The parameter $a$ represents the normalization factor and the additional contribution (1 $a)^{2} / \tilde{\sigma}^{2}$ is related to the systematic uncertainty of the overall neutrino flux at the source $(\tilde{\sigma}=0.2)$. This systematic uncertainty [54] plays an important role in the correct estimation of the sensitivity of the experiment especially when the shape of $\nu_{\mu} \mathrm{CC}$ events spectrum is not changed by the decoherence effects (i.e. exponents independent on energy).

For the best-fit values [36] of the atmospheric neutrino parameters, we used

$$
\Delta m^{2}=2.5 \times 10^{-3} \mathrm{eV}^{2} ; \quad \theta_{23}=45^{\circ} .
$$




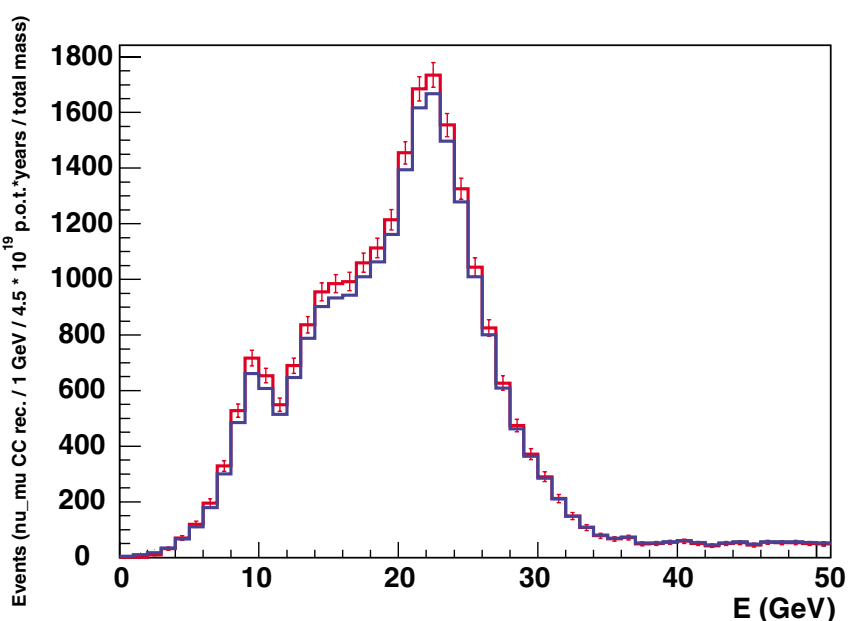

FIG. 2 (color online). The number of reconstructed $\nu_{\mu} \mathrm{CC}$ events in OPERA as a function of the neutrino energy with (blue line) and without (red line with error bars) QG decoherence effect included in case of inversely proportional dependence on neutrino energy. $3 \sigma$ difference between the expected and QG disturbed spectra is shown.

The $3 \sigma$ sensitivity on the damping parameters is found by applying a cut on the value of the $\chi^{2}$ of 9 and 11.83 , respectively, for 1 d.o.f. and 2 d.o.f.

As the CNGS beam is designed to observe $\nu_{\tau}$, neutrinos will have a high energy with a mean value of about $17 \mathrm{GeV}$. This represents an advantage since it makes the exponential damping factors more pronounced for some cases described in the previous section. For the OPERA experiment, the systematic uncertainty in the muon detection efficiency is negligible compared to the statistical uncertainties, therefore the error $\sigma_{i}$ used in our analysis [Eq. (51)] represents the statistical error only.

To generate the expected neutrino spectra of the CNGS beam measured by the OPERA experiment, we used a fast simulation algorithm described in [55] (see also Appendix A for details). We present in Fig. 2 a typical simulated spectrum of the expected number of $\mu$ events including the effects of decoherence (for the case of an inversely proportional dependence on neutrino energy) as a subdominant suppression of the probability inferred from the atmospheric neutrino experiment [36].

Our results for the sensitivity of CNGS to oneparametric decoherence damping exponentials in $P_{\nu_{\mu} \rightarrow \nu_{\tau}}$ are summarized in the second column of Table I. Also, for two parametric fits (45) and (48), the $3 \sigma$ C.L. sensitivity contours are presented in Fig. 3.

Contrary to the OPERA experiment, the T2K experiment was designed to observe $\nu_{e}$ and the mean energy is much lower: the maximum of oscillation at the given baseline of $295 \mathrm{~km}$ corresponds to a neutrino energy of about $600 \mathrm{MeV}$ and a narrow spectra at the selected energy will be obtained using the so-called off-axis technique [56]. The spectrum covers the region of the first maximum of oscillation and this is a region where the QG effects could be easily observed due to the small number of $\nu_{\mu} \mathrm{CC}$ events expected in case of no QG damping exponents, as can be seen in Fig. 4.

The neutrino production at J-PARC beam is simulated in GEANT environment, which takes into account the whole focusing system (horn and reflectors), target, and decay tunnel at J-PARC. Protons are generated on target and through the decay of parent pions and kaons the probability of neutrino at a selected location is calculated and the spectra is obtained. We use the reconstructed neutrino energy for single-Cherenkov-ring muon quasielastic $(\mathrm{QE})$ and non-QE events. Of course, here the energy resolution plays an important role: for this reason we introduced an energy smearing effect of $20 \%$ in our analysis. This value takes into account the different energy resolution for the two kinds of events and the fact that QE events are the majority of the of muon neutrino events in the detector.

Our results obtained using the same way of analysis quantified by (50) and (51) for the sensitivity of T2K to one-parametric decoherence damping exponentials in $P_{\nu_{\mu} \rightarrow \nu_{\tau}}$ are summarized in the third column of Table I. Also, for two parametric fits (45) and (48), the $3 \sigma$ C.L.

TABLE I. Expected sensitivity limits at CNGS, T2K, and T2KK to one-parametric neutrino decoherence for Lindblad type and gravitational MSW (stochastic metric fluctuation) like operators. These results are obtained for the "true" values of the oscillation parameters fixed at $\Delta m^{2}=2.5 \times 10^{-3} \mathrm{eV}^{2}$ and $\theta_{23}=45^{\circ}$ [36].

\begin{tabular}{lccc}
\hline \hline Lindblad-type mapping operators & CNGS & T2K & T2KK \\
\hline$\gamma_{0}[\mathrm{eV}] ;([\mathrm{GeV}])$ & $2 \times 10^{-13} ;\left(2 \times 10^{-22}\right)$ & $2.4 \times 10^{-14} ;\left(2.4 \times 10^{-23}\right)$ & $1.7 \times 10^{-14} ;\left(1.7 \times 10^{-23}\right)$ \\
$\gamma_{-1}^{2}\left[\mathrm{eV}^{2}\right] ;\left(\left[\mathrm{GeV}^{2}\right]\right)$ & $9.7 \times 10^{-4} ;\left(9.7 \times 10^{-22}\right)$ & $3.1 \times 10^{-5} ;\left(3.1 \times 10^{-23}\right)$ & $6.5 \times 10^{-5} ;\left(6.5 \times 10^{-23}\right)$ \\
$\gamma_{2}\left[\mathrm{eV}^{-1}\right] ;\left(\left[\mathrm{GeV}^{-1}\right]\right)$ & $4.3 \times 10^{-35} ;\left(4.3 \times 10^{-26}\right)$ & $1.7 \times 10^{-32} ;\left(1.7 \times 10^{-23}\right)$ & $3.5 \times 10^{-33} ;\left(3.5 \times 10^{-24}\right)$ \\
\hline Gravitational MSW (stochastic) effects & $\mathrm{CNGS}$ & $\mathrm{T} 2 \mathrm{~K}$ & $\mathrm{~T} 2 \mathrm{KK}$ \\
\hline$\alpha^{2}$ & $4.3 \times 10^{-13} \mathrm{eV}$ & $4.6 \times 10^{-14} \mathrm{eV}$ & $3.5 \times 10^{-14} \mathrm{eV}$ \\
$\alpha_{1}^{2}$ & $1.1 \times 10^{-25} \mathrm{eV}^{2}$ & $3.2 \times 10^{-26} \mathrm{eV}^{2}$ & $6.7 \times 10^{-27} \mathrm{eV}^{2}$ \\
$\beta^{2}$ & $3.6 \times 10^{-24}$ & $5.6 \times 10^{-23}$ & $1.7 \times 10^{-23}$ \\
$\beta_{2}^{2}$ & $9.8 \times 10^{-37} \mathrm{eV}^{-36}$ & $4 \times 10^{-35} \mathrm{eV}^{-3}$ & $3.1 \times 10^{-36} \mathrm{eV}^{-33}$ \\
$\beta_{1}^{2}$ & $8.8 \times 10^{-35} \mathrm{eV}^{-1}$ & $3.5 \times 10^{-32} \mathrm{eV}^{-1}$ & $7.2 \times 10^{-33} \mathrm{eV}^{-1}$ \\
\hline \hline
\end{tabular}



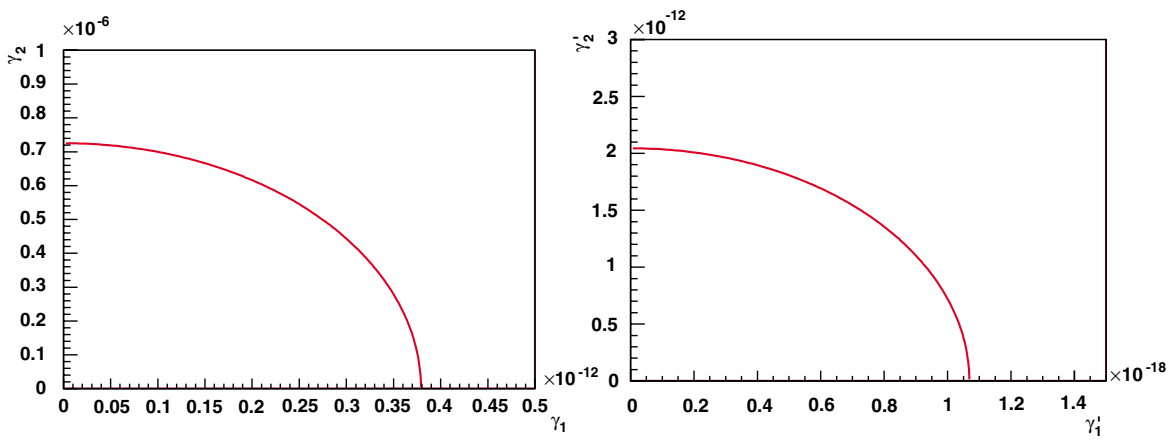

FIG. 3 (color online). The expected CNGS sensitivity contour at $3 \sigma$ C.L., with two decoherence parameters contributing to the combined time evolution of the gravitational MSW effect (with stochastic metric fluctuations), calculated for damping with no energy dependence (left panel) and damping proportional to the neutrino energy (right panel).

sensitivity contours are presented in Fig. 5. Contrary to CNGS, the J-PARC facility is equipped with a near detec-

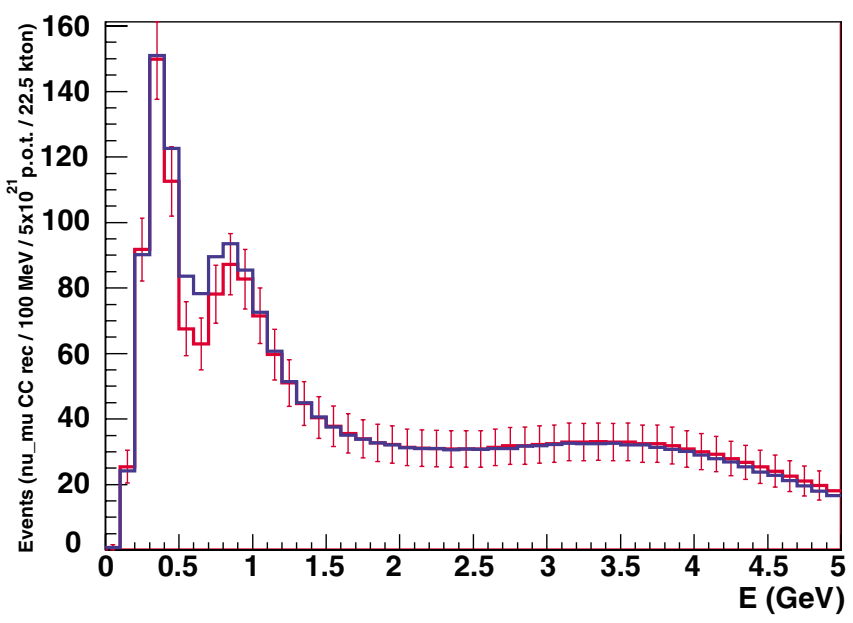

FIG. 4 (color online). The number of reconstructed $\nu_{\mu} \mathrm{CC}$ events in $\mathrm{T} 2 \mathrm{~K}$ as a function of the neutrino energy with (blue line) and without (red line with error bars) QG decoherence effect included in the case of inversely proportional dependence on neutrino energy. $3 \sigma$ difference between the expected and QG disturbed spectra is shown. tor which measures the unoscillated muon spectrum with $5 \%$ uncertainty in the absolute normalization of the overall flux. However, to be conservative, we obtain our results in the $3 \mathrm{rd}$ and 4 th columns of Table I under the assumption of $20 \%$ uncertainty in the overall normalization of the spectrum. Since the main effect is related to the maximal oscillation point in the spectrum, the overall normalization is not as critical as in the case of CNGS fit.

The T2K experiment yields a better limit on the damping parameters only in the case where the effect has no energy dependence or contains inversely proportional to the neutrino-energy exponent, as expected given the lowenergy spectrum. In all the other cases, the dependence on the baseline disfavors the short baseline of T2K with respect to OPERA.

Another possibility to observe the effect on the T2K neutrino beam is to select a longer baseline, namely, to locate the detector at about $1000 \mathrm{~km}$ in Korea. Studies of beam upgrades and a large liquid argon detector of 100 kton in Korea were carried out [57] in the framework of $C P$ violation discovery. We considered this option, called $\mathrm{T} 2 \mathrm{KK}$, and studied the possibility to constrain damping parameters in this case. The proposed upgrade at $4 \mathrm{MW}$ of the beam was taken into account which results
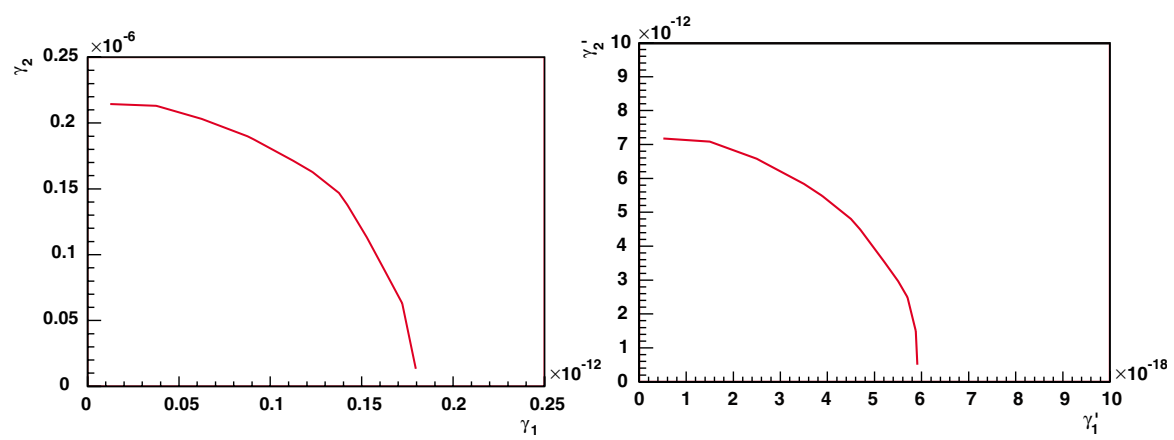

FIG. 5 (color online). The expected T2K sensitivity contour at $3 \sigma$ C.L., with two decoherence parameters contributing to the combined time evolution of the gravitational MSW effect (with stochastic metric fluctuations), calculated for damping with no energy dependence (left panel) and damping proportional to the neutrino energy (right panel). 
into $7 \times 10^{21}$ p.o.t. per year and a running time of 4 years was envisaged. The efficiency $\epsilon_{\mu \mu}$ for the detector is assumed to be $95 \%$ and an energy smearing of $15 \%$ is taken into account.

Our results for the sensitivity of T2KK to oneparametric decoherence damping exponentials in $P_{\nu_{\mu} \rightarrow \nu_{\tau}}$ are summarized in the fourth column of Table I. This configuration yields better results than the $\mathrm{T} 2 \mathrm{~K}$ experiment and results comparable to the OPERA experiment.

All bounds obtained in Table I are evaluated at the bestfit oscillation parameters given by (52). In Appendix B we present the behavior of the expected sensitivities obtained for simple one-parametric damping exponents as functions of oscillation parameters that are varied in the vicinity of the best-fit values (52). The sensitivities given in Table I are very close to the worst ones, obtained by allowing the oscillation parameters to vary (see Figs. 6 and 7).
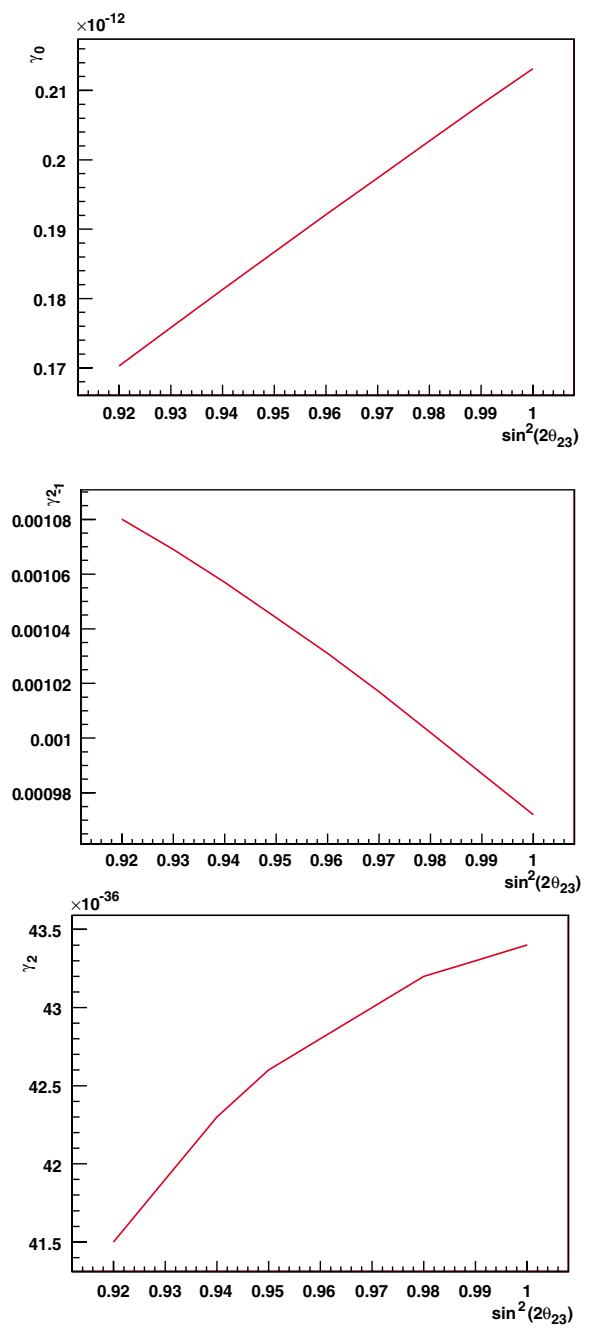

\section{DISCUSSION AND OUTLOOK}

It is instructive to compare the sensitivity limits presented in Table I with those derived from the analysis of atmospheric neutrino data [19] obtained at SuperKamiokande and $\mathrm{K} 2 \mathrm{~K}$ experiments. One can transform the limits on the Lindblad-type operators presented in Table I to the notations (32) of [19] using the following transformations:

$$
\begin{aligned}
& \gamma_{\mathrm{Lnb}}=\gamma_{0}[\mathrm{GeV}], \quad n=0 \\
& \gamma_{\mathrm{Lnb}}=\gamma_{2}\left[\mathrm{GeV}^{-1}\right] \times\left(\mathrm{GeV}^{2}\right), \quad n=2 \\
& \gamma_{\mathrm{Lnb}}=\gamma_{-1}^{2}\left[\mathrm{GeV}^{2}\right] /(\mathrm{GeV}), \quad n=-1,
\end{aligned}
$$

so that the numbers of Table I in parentheses can be directly compared with the bounds (32) and (33). In particular, the bound obtained in [19] [see for details (32)] at
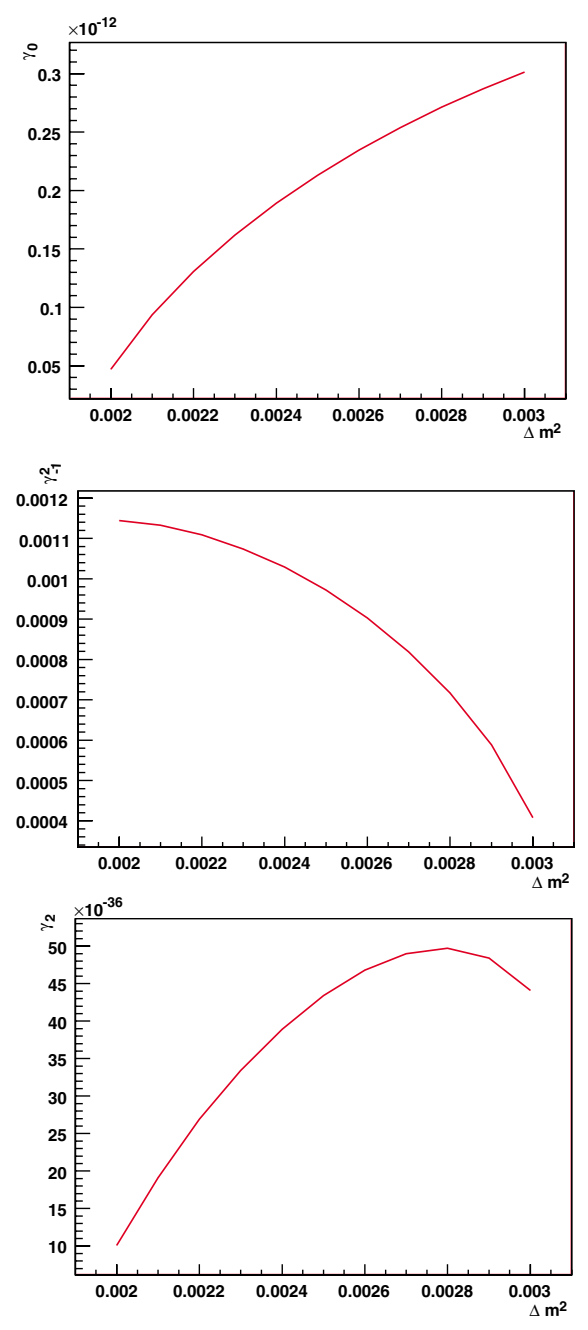

FIG. 6 (color online). The sensitivity of CNGS to probabilities corrected for one-parametric decoherence effects as functions of the true values of $\theta_{23}$ (left panels) and $\Delta m_{23}$ (right panels). The input value for the mass difference in the left panels is $\Delta m^{2}=$ $2.5 \times 10^{-3} \mathrm{eV}^{2}$, while for the right panels $\theta_{23}=45^{\circ}$. Three cases of damping exponents are considered with no energy dependence (top), inverse-energy dependence (middle), and quadratic energy dependence (bottom). 

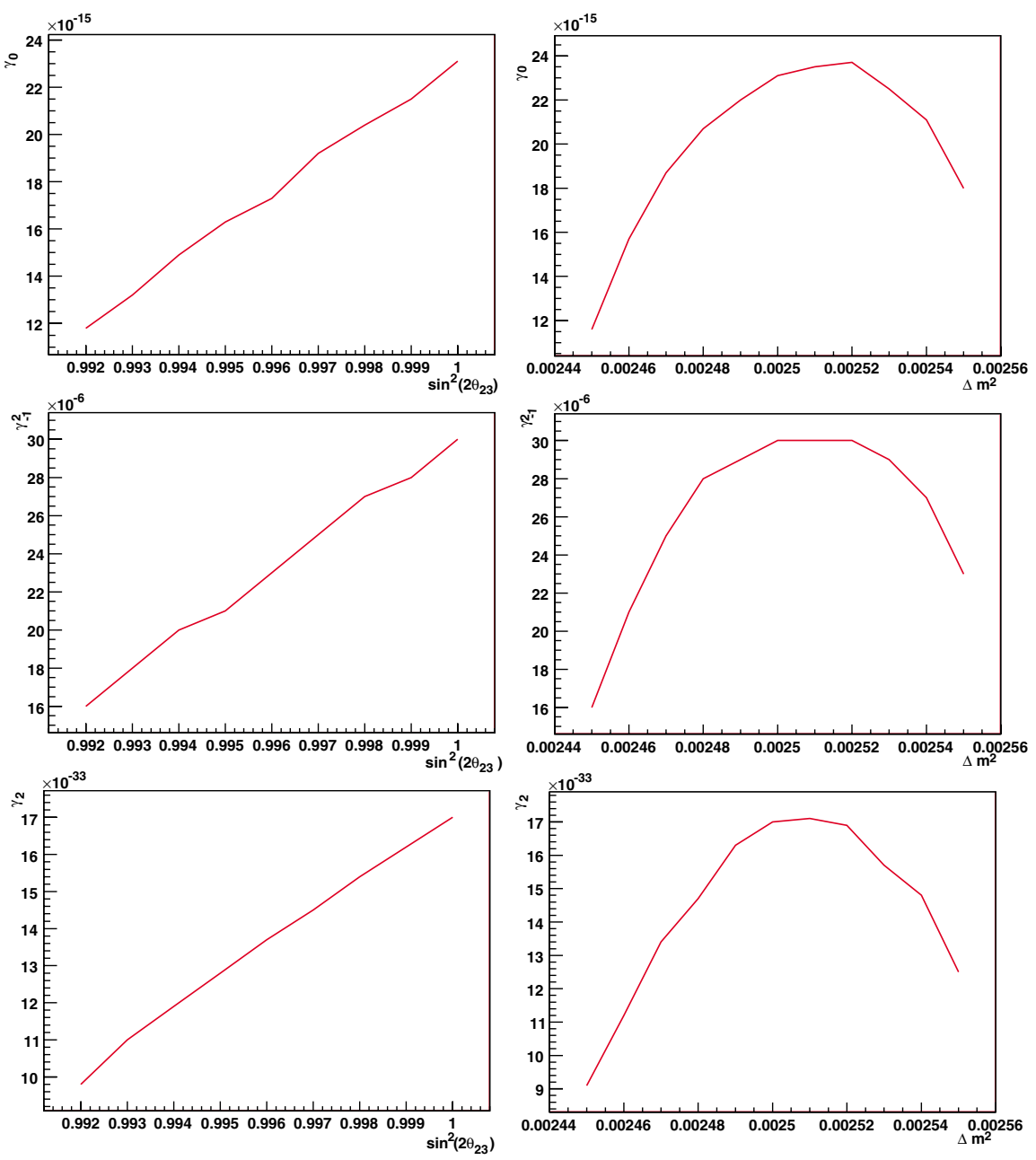

FIG. 7 (color online). The same as in Fig. 6 calculated for the sensitivity of T2K.

95\% C.L. on the Lindblad-type operators with no energy dependence is close to the sensitivity estimated in our analysis in case of $\mathrm{T} 2 \mathrm{~K}$ and $\mathrm{T} 2 \mathrm{KK}$ simulations. Although, the CNGS estimation is about an order of magnitude weaker, one should stress that the current limit is given at $99 \%$ C.L. under the assumption of the most conservative level of the uncertainty of the overall neutrino flux at the source. The bound on the inverse-energy dependence given in [19] (32) is close to the current CNGS estimates. T2K and T2KK demonstrate an improvement. In spite of the fact that the Super-Kamiokande data contains neutrinos of energies up to $\sim \mathrm{TeV}$, the sensitivity one obtains at CNGS to the energy-squared dependent decoherence is close, within an order of magnitude, to the bound (32) imposed by atmospheric neutrinos and surpasses $\mathrm{T} 2 \mathrm{~K}$ and $\mathrm{T} 2 \mathrm{KK}$ sensitivity bounds by $\approx 3$ and $\approx$ 2 orders of magnitude, respectively. The much less uncertain systematics of CNGS compared to the atmospheric neutrino data will make the expected bound more robust as soon as the upcoming data from OPERA will be analyzed. Moreover, our results are also competitive with the sensitivity to the same Lindblad operators estimated in [58] for
ANTARES neutrino telescope, which is supposed to operate at neutrino energies much higher than CNGS and JPARC experiments [59].

Assuming that the decoherence phenomena affect all particles in the same way, which however is by no means certain, one might compare the results of our analysis with bounds obtained using the neutral kaon system [60]. The comparison could be done for the constant (no-energy dependence) Lindblad decoherence model. The main bound in [60] in such a case reads $\gamma_{0} \leq 4.1 \times 10^{-12} \mathrm{eV}$, thus being about 2 orders of magnitude weaker than the sensitivity forecasted in the present paper.

Finally, we compare the estimated sensitivity with the bounds obtained in [27] using solar + KamLAND data. In principle, as in the case of the neutral kaon system, a direct comparison is impossible, since the parameters investigated here for the $\nu_{\mu} \rightarrow \nu_{\tau}$ channel need not be the same for the $\nu_{e} \rightarrow \nu_{\mu}$ channel. However, again, if these parameters are assumed to be roughly of equal size, then one can see that the estimates of [27] (33), which win essentially over the CNGS, T2K and T2KK sensitivities only for the case of inverse-energy dependent decoherence, which 
strongly favors low neutrino energies [e.g. the case of Cauchy-Lorentz stochastic space-time foam models of [37] (29), for which the current limit would bound, on account of (40), the scale parameter $\xi$ of the distribution (27) to: $\xi<5 \times 10^{-3}$ for neutrino-mass differences [27] $\left.\left|\mathrm{m}_{e}^{2}-\mathrm{m}_{\mu}^{2}\right|=(7.92 \pm 0.71) \times 10^{-5} \mathrm{eV}^{2}\right]$. For the completeness, we mention that, our best expected bound on the inverse-energy decoherence will imply, according to (30) the bound on the $\nu_{\mu}$ life time $\tau_{\nu_{\mu}} / m_{\nu_{\mu}}>3 \times$ $10^{22} \mathrm{GeV}^{-2}$.

The precise energy and length dependence of the damping factors is an essential step in order to determine the microscopic origin of the induced decoherence and disentangle genuine new physics effects from conventional effects, which as we have seen in Sec. III may also contribute to decoherencelike damping. Some genuine quantumgravity effects, such as the MSW-like effect induced by stochastic fluctuations of the space-time, are expected to increase in general with the energy of the probe, as a result of backreaction effected on space-time geometry, in contrast to ordinary-matter-induced "fake" $C P T$ violation and "decoherence-looking" effects, which decrease with the energy of the probe [50]. At present, as one can see from the Sec. III, the sensitivity of the experiments is not sufficient to unambiguously determine the microscopic origin of the decoherence effects, but according to our estimations of the most plausible energy-length dependencies for the MSW-like decoherence the sensitivity of CNGS and T2K will improve the current limits by at least 2 orders of magnitude, and one would arrive at definite conclusions on this important issue. Thus, phenomenological analyses like ours are of value and should be actively pursued when the data from OPERA and T2K will become available. When the present paper was finished, we became aware of a similar analysis [61] performed for J-PARC experiments which agrees with our results concerning T2K and T2KK.

In general, the characteristic energy dependencies of damping features are very interesting to search for physics beyond the standard model. In some cases, such damping signatures could be compensated by a shift of the neutrino oscillation parameters, which means that, given such a damping effect, it is quite possible to obtain an erroneous determination of these parameters. However, if the damping effects are strong enough, then an establishment of effects beyond the standard neutrino oscillation scenario will be possible. Once such a damping effect is established, it will be very interesting to know from which nonstandard mechanism it actually arises. Given this identification problem, we have found quite a low sensitivity for the models with inverse-energy dependence in the exponent, which means that the damping effects of such kind are strongly correlated with the standard neutrino oscillation parameters, i.e., it is difficult to distinguish them from small adjustments in the oscillation parameters at CNGS and T2K. However, damping signatures similar to energy dependence or energy dependence squared can be very easily disentangled from the standard oscillations, but it is difficult to distinguish them from each other. Concerning different time dependencies in the exponents including the combined signatures we have analyzed, it can, in principle, be resolved if there are two baselines, as applied, for example, in [61], and all the other parameters are known. Also, for a specific model, there may be relations among different $\gamma$ 's in (45) and (48) like fits that actually imply much fewer independent parameters.

\section{ACKNOWLEDGMENTS}

The work of N.E. M. and S. S. is partially supported by the European Union through the Marie Curie Research and Training Network UniverseNet MRTN-2006-035863.

\section{APPENDIX A: SIMULATION OF NEUTRINO BEAMS}

A wideband neutrino beam is produced from the decay of mesons, mostly $\pi$ 's and $K$ 's. Mesons are created by the interaction of a proton beam into a needle shaped target, they are sign-selected and focused in the forward direction by two large acceptance magnetic coaxial lenses, conventionally called at CNGS (CERN) horn and reflector, and finally they are let to decay into an evacuated tunnel pointing toward the detector position.

In the case of positive charge selection, the beam content is mostly $\nu_{\mu}$ from the decay of $\pi^{+}$and $K^{+}$. Small contaminations of $\bar{\nu}_{\mu}$ (from the defocused $\pi^{-}$and $K^{-}$) and $\nu_{e}$ (from three-body decay of $K^{\prime}$ 's and $\mu$ 's) are present at the level of few percent.

The neutrino fluxes for such a kind of beam are relatively easy to predict [55] once the secondary meson spectra are known, because the meson decay kinematics is well understood and the geometry of the decay tunnel is quite simple.

Uncertainty in the estimation of the neutrino fluxes could arise because secondary mesons are selected over a wide momentum range and over a wide angular acceptance ( $\simeq 20 \mathrm{mrad})$.

Reinteractions of secondary mesons in the target and downstream material contribute to reduce the neutrino fluxes and increase the uncertainty in the calculations (mainly for the wrong sign and wrong flavor contaminations). They are generally minimized using a target made of a number thin rods of low $Z$ material interleaved with empty spaces (to let the secondary mesons exit the target without traversing too much material). In addition, the amount of material downstream of the target (i.e. horn and reflector conductor thickness) is kept to the minimum.

The parametrization of the secondary meson production from protons onto a thin target, proposed in [55], is thus well suited to be used in neutrino beam simulations both because it extends its prediction over a wide range of 
longitudinal and transverse momenta and also because the small fraction of tertiary production from reinteractions in the target and downstream material can be accounted for with the approximations described in [55]. A comparison of the neutrino flux prediction based on the parametrization of [55] with some measured spectra is thus an effective estimator of the quality of the secondary mesons parametrization. For this purpose, the parametrization [55] has been coupled with a neutrino beam simulation program to be able to provide rapid and accurate predictions of neutrino spectra at any distance (i.e. short and long base line). The comparison has been performed both with already published data (CHARM II) and with predictions for the future CNGS long-baseline neutrino beam generated with GEANT and/or FLUKA based Monte Carlo programs.

The resulting code [62] is a stand-alone application developed on the basis of parametrization [55] that allows one to vary and optimize all elements and the geometry (in 3D) of the beam line providing the results in terms of neutrino spectra and distributions at large distance with high statistics and in short time.

The underlying idea is that in order to produce rapidly a neutrino spectrum at large distance over a small solid angle at CNGS beam, one has to force all the mesons to decay emitting a neutrino, and force all neutrinos to cross the detector volume. A weight is then assigned to each neutrino, proportional to the probability that this process actually happened. In practice, this method is implemented by subdividing the simulation into four subsequent steps, as described in detail in [55]. These steps include mesons production processes along target, meson tracking in the neutrino beam line, neutrino production processes from mesons, and neutrino production from muons. The weights associated with every step are described in detail in [55].

\section{APPENDIX B: CORRELATIONS BETWEEN DAMPING EXPONENTS AND STANDARD PARAMETERS}

Here we present the results of fitting of the simulated spectra, Figs. 2 and 4, with varying oscillation parameters to the simple one-parametric decoherence models reported in the upper half of Table I for CNGS and J-PARC's T2K option.

The contours in Figs. 6 and 7 were obtained varying the atmospheric oscillation parameters (either $\Delta m^{2}$ at fixed input value of $\theta_{23}=45^{\circ}$ or $\theta_{23}$ at $\Delta m^{2}=2.5 \times$ $10^{-3} \mathrm{eV}^{2}$ ) for the calculation of the spectra assuming decoherence, whereas the true spectra was simulated for the best-fit atmospheric parameters (52).
[1] See, for instance, J. A. Wheeler and K. Ford, Geons, Black Holes, and Quantum Foam: A Life in Physics (Norton, New York, USA, 1998), p. 380, and references therein.

[2] S. W. Hawking, Commun. Math. Phys. 87, 395 (1982).

[3] J. M. Maldacena, J. High Energy Phys. 04 (2003) 021.

[4] S. W. Hawking, Phys. Rev. D 72, 084013 (2005).

[5] R. Brustein, M. B. Einhorn, and A. Yarom, J. High Energy Phys. 04 (2007) 086.

[6] J. R. Ellis, N. E. Mavromatos, and D. V. Nanopoulos, Int. J. Mod. Phys. A 13, 1059 (1998); Phys. Rev. D 62, 084019 (2000).

[7] F. Benatti and R. Floreanini, Ann. Phys. (N.Y.) 273, 58 (1999).

[8] J.R. Ellis, J.S. Hagelin, D. V. Nanopoulos, and M. Srednicki, Nucl. Phys. B241, 381 (1984).

[9] T. Banks, L. Susskind, and M.E. Peskin, Nucl. Phys. B244, 125 (1984).

[10] G. Lindblad, Commun. Math. Phys. 48, 119 (1976); R. Alicki and K. Lendi, Quantum Dynamical Semigroups and Applications, Lect. Notes Phys. (Springer-Verlag, Berlin, 1987), Vol. 286; Quantum Dynamical Semigroups and Applications, Lect. Notes Phys. (Springer-Verlag, Berlin, 2007), Vol. 717.

[11] V. Gorini, A. Kossakowski, and E. C. G. Sudarshan, J. Math. Phys. (N.Y.) 17, 821 (1976).

[12] N. Mavromatos and S. Sarkar, Phys. Rev. D 72, 065016 (2005).
[13] For a review, see N.E. Mavromatos, Planck Scale Effects in Astrophysics and Cosmology, Lect. Notes Phys. (Springer-Verlag, Berlin, 2005), Vols. 669, p. 245, and references therein.

[14] J. R. Ellis, N. E. Mavromatos, and D. V. Nanopoulos, Phys. Lett. B 293, 37 (1992); Special issue on A Microscopic Liouville Arrow of Time, edited by C. Castro and M. S. El Naschie [J. Chaos Solitons Fractals 10, 345 (1999)].

[15] R. Brustein, M. B. Einhorn, and A. Yarom, J. High Energy Phys. 01 (2006) 098.

[16] L. J. Garay, Int. J. Theor. Phys. 41, 2047 (2002); Int. J. Mod. Phys. A 14, 4079 (1999).

[17] B. L. Hu and E. Verdaguer, Classical Quantum Gravity 20, R1 (2003); Living Rev. Relativity 7, 3 (2004).

[18] J. R. Ellis, N. E. Mavromatos, and D. V. Nanopoulos, Phys. Rev. D 63, 024024 (2000).

[19] E. Lisi, A. Marrone, and D. Montanino, Phys. Rev. Lett. 85, 1166 (2000).

[20] G. Barenboim and N.E. Mavromatos, Phys. Rev. D 70, 093015 (2004).

[21] G. Barenboim and N.E. Mavromatos, J. High Energy Phys. 01 (2005) 034.

[22] F. Benatti and R. Floreanini, Phys. Rev. D 64, 085015 (2001).

[23] F. Benatti and R. Floreanini, J. High Energy Phys. 02 (2000) 032

[24] R. Brustein, D. Eichler, and S. Foffa, Phys. Rev. D 65, 
105006 (2002).

[25] G. Barenboim, N.E. Mavromatos, S. Sarkar, and A. Waldron-Lauda, Nucl. Phys. B758, 90 (2006).

[26] G. L. Fogli, E. Lisi, A. Marrone, and D. Montanino, Phys. Rev. D 67, 093006 (2003).

[27] G. L. Fogli, E. Lisi, A. Marrone, D. Montanino, and A. Palazzo, Phys. Rev. D 76, 033006 (2007).

[28] D. Hooper, D. Morgan, and E. Winstanley, Phys. Rev. D 72, 065009 (2005); Phys. Lett. B 609, 206 (2005).

[29] L. A. Anchordoqui, H. Goldberg, M. C. Gonzalez-Garcia, F. Halzen, D. Hooper, S. Sarkar, and T. J. Weiler, Phys. Rev. D 72, 065019 (2005).

[30] A. M. Gago, E. M. Santos, W. J.C. Teves, and R. Zukanovich Funchal, arXiv:hep-ph/0208166.

[31] A. Aguilar et al. (LSND Collaboration), Phys. Rev. D 64, 112007 (2001); G. Drexlin, Nucl. Phys. B, Proc. Suppl. 118, 146 (2003).

[32] A. A. Aguilar-Arevalo et al. (MiniBooNE Collaboration), Phys. Rev. Lett. 98, 231801 (2007).

[33] M. Maltoni and T. Schwetz, Phys. Rev. D 76, 093005 (2007).

[34] J. R. Ellis, N. E. Mavromatos, and D. V. Nanopoulos, Phys. Lett. B 293, 142 (1992); J. R. Ellis, J. L. Lopez, N.E. Mavromatos, and D. V. Nanopoulos, Phys. Rev. D 53, 3846 (1996); P. Huet and M. E. Peskin, Nucl. Phys. B434, 3 (1995); F. Benatti and R. Floreanini, Phys. Lett. B 468, 287 (1999).

[35] T. Araki et al. (KamLAND Collaboration), Phys. Rev. Lett. 94, 081801 (2005).

[36] Y. Ashie et al. (Super-Kamiokande Collaboration), Phys. Rev. Lett. 93, 101801 (2004).

[37] J. Alexandre, K. Farakos, N.E. Mavromatos, and P. Pasipoularides, arXiv:0712.1779.

[38] J. R. Ellis, N. E. Mavromatos, and M. Westmuckett, Phys. Rev. D 70, 044036 (2004); 71, 106006 (2005).

[39] D. Giulini, E. Joos, C. Kiefer, J. Kupsch, I.-O Stamatescu, and H.D. Zeh, Decoherence and the Appearance of a Classical World in Quantum Theory (Springer, New York, 1996).

[40] R. M. Wald, Phys. Rev. D 21, 2742 (1980).

[41] M. Srednicki, Phys. Rev. Lett. 71, 666 (1993).

[42] L. Wolfenstein, Phys. Rev. D 17, 2369 (1978).

[43] S. P. Mikheev and A. Y. Smirnov, Yad. Fiz. 42, 1441
(1985) [Sov. J. Nucl. Phys. 42, 913 (1985)].

[44] F. N. Loreti and A. B. Balantekin, Phys. Rev. D 50, 4762 (1994); E. Torrente-Lujan, arXiv:hep-ph/0210037.

[45] N.E. Mavromatos and S. Sarkar, Phys. Rev. D 74, 036007 (2006).

[46] S. Gao, Phys. Rev. D 68, 044028 (2003); for the charge scalar particle case, see H.b. Zhang, Z.j. Cao, and C.s. Gao, Commun. Theor. Phys. 41, 385 (2004).

[47] G. Lifschytz, J. High Energy Phys. 09 (2004) 009; J. High Energy Phys. 08 (2004) 059.

[48] T. Ohlsson, Phys. Lett. B 502, 159 (2001).

[49] M. Blennow, T. Ohlsson, and W. Winter, J. High Energy Phys. 06 (2005) 049; M. Jacobson and T. Ohlsson, Phys. Rev. D 69, 013003 (2004).

[50] M. Blennow, T. Ohlsson, and W. Winter, J. High Energy Phys. 06 (2005) 049.

[51] M. Furusaka et al. (Joint Project team of JAERI and KEK Collaboration), Report No. KEK-REPORT-99-4; Y. Itow et al., arXiv:hep-ex/0106019.

[52] R. Acquafredda et al. (OPERA Collaboration), New J. Phys. 8, 303 (2006).

[53] Y. Fukuda et al. (Super-Kamiokande Collaboration), Phys. Rev. Lett. 81, 1562 (1998).

[54] For our estimations, we take the most conservative $20 \%$ uncertainty of the overal flux normalization.

[55] M. Bonesini, A. Marchionni, F. Pietropaolo, and T. Tabarelli de Fatis, Eur. Phys. J. C 20, 13 (2001).

[56] K. T. McDonald, arXiv:hep-ex/0111033.

[57] A. Meregaglia and A. Rubbia, arXiv:0801.4035.

[58] D. Morgan, E. Winstanley, J. Brunner, and L.F. Thompson, Astropart. Phys. 25, 311 (2006).

[59] Although the sensitivity to the energy squired depending damping exponent obtained in [58] is very close to our estimations for CNGS, it is unclear why the authors of [58] claim a remarkable improvement of this bound relative to the atmospheric bound (32).

[60] J. Ellis et al. (CPLEAR collaboration), Phys. Lett. B 364, 239 (1995).

[61] N. C. Ribeiro, T. Kajita, P. Ko, H. Minakata, S. Nakayama, and H. Nunokawa, arXiv:0712.4314.

[62] The source code is available at http://castore.mib.infn.it/ BMPT. 